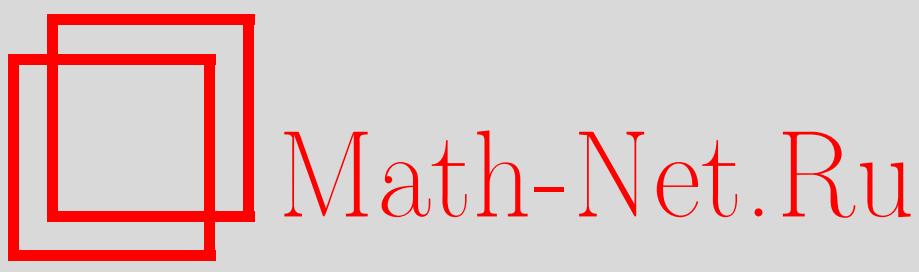

Д. В. Жданович, Внешние автоморфизмы локально простых алгебр Ли, Матем. сб., 1997, том 188, номер 9, 31-54

DOI: https://doi.org/10.4213/sm256

Использование Общероссийского математического портала Math-Net.Ru подразумевает, что вы прочитали и согласны с пользовательским соглашением http://www . mathnet.ru/rus/agreement

Параметры загрузки:

IP: 3.85 .7 .115

26 апреля 2023 г., $15: 50: 37$ 
УДК 512.554

\title{
Д.В. Жданович
}

\section{Внешние автоморфизмы локально простых алгебр Ли}

\begin{abstract}
В статье рассматриваются группы автоморфизмов локально простых алгебр Ли над полем $\mathbb{C}$. Выясняется, что существует естественное определение группы внутренних автоморфизмов таких алгебр Ли, которая является нормальной подгруппой в группе автоморфизмов. Следовательно, можно определить группу внешних автоморфизмов локально простой алгебры Ли. Показано, что, в отличие от конечномерного случая, группа внешних автоморфизмов не обязана быть конечной и в отдельных случаях эта группа вычислена полностью.

Библиографол: 6 названий.
\end{abstract}

\section{§1. Введение}

ОПРЕДЕЛЕНИЕ 1.1. Алгебра Ли g называется локально простой, если для любого конечного подмножества $Y \subset \mathrm{g}$, сушествует простая конечномерная подалгебра Ли $g_{Y}$, которая содержит это множество.

Если локально простая алгебра Ли g не более чем счетномерна, то она является прямым пределом индуктивной системы следующего вида

$$
\mathrm{g}_{1} \stackrel{\varphi_{1}}{\longrightarrow} \mathrm{g}_{2} \stackrel{\varphi_{2}}{\longrightarrow} \mathrm{g}_{3} \rightarrow \cdots \rightarrow \mathrm{g}_{i} \stackrel{\varphi_{i}}{\longrightarrow} \mathrm{g}_{i+1} \rightarrow \cdots,
$$

где $\mathrm{g}_{i}-$ это конечномерная простая алгебра Ли для любого $i \geqslant 1$.

В настоящей статье рассматриваются не более чем счетномерные локально простые алгебры Ли над полем комплексных чисел $\mathbb{C}$. Интерес к таким алгебрам возник после работы [1], в которой было показано, что запас таких алгебр довольно большой.

В данной работе исследуются групшы автоморфизмов локально простых алгебр Ли. Представление локально простой алгебры Ли в виде прямого предела индуктивной системы конечномерных простых алгебр Ли позволяет сразу получить автоморфизмы, которые происходят из связи теории групп Ли и теории конечномерњых алгебр Ли (см. замечание 2). Такие автоморфизмы естественно назвать внутренними.

Как обычно, внутренние автоморфизмы образуют нормальную подгруппу $\operatorname{Int}(\mathrm{g})$ в группе $\operatorname{Aut}(\mathrm{g})$ и, следовательно, определена факторгруппа $\operatorname{Aut}(\mathrm{g}) / \operatorname{Int}(\mathrm{g})$, которая названа группой внешних автоморфизмов.

Вычисление групшы внешних автоморфизмов в конечномерном случае не составляет труда. Она изоморфна группе автоморфизмов соответствуюшей схемы Дынкина [2]. В обшем счетномерном случае задача вычисления группы внешних автоморфизмов, по всей видимости, является трудно выполнимой. 
В работе [3] среди всех локально полупростых алгебр Ли был выделен некоторый класс алгебр Ли, названный диагональным, и приведены достаточно убедительные доводы в пользу его содержательности.

Пусть алгебра Ли является прямым пределом индуктивной системы следующего вида

$$
\operatorname{sl}_{n_{1}}(\mathbb{C}) \stackrel{\varphi_{1}}{\longrightarrow} \operatorname{sl}_{n_{2}}(\mathbb{C}) \stackrel{\varphi_{2}}{\longrightarrow} \operatorname{sl}_{n_{3}}(\mathbb{C}) \longrightarrow \cdots \longrightarrow \operatorname{sl}_{n_{i}}(\mathbb{C}) \stackrel{\varphi_{i}}{\longrightarrow} \operatorname{sl}_{n_{i+1}}(\mathbb{C}) \longrightarrow \cdots
$$

Если в этой индуктивной системе алгебры Ли $\mathrm{sl}_{n_{i}}(\mathbb{C})$ можно реализовать в виде матриц размера $n_{i} \times n_{i}$ с нулевьм следом так, что вложения $\varphi_{i}$ примут вид

$$
\varphi_{i}(A)=\left(\begin{array}{ccccccccc}
A & \cdots & 0 & 0 & \cdots & 0 & 0 & \cdots & 0 \\
\vdots & \ddots & \vdots & \vdots & & \vdots & \vdots & & \vdots \\
0 & \cdots & A & 0 & \cdots & 0 & 0 & \cdots & 0 \\
0 & \cdots & 0 & -A^{T} & \cdots & 0 & 0 & \cdots & 0 \\
\vdots & & \vdots & \vdots & \ddots & \vdots & \vdots & & \vdots \\
0 & \cdots & 0 & 0 & \cdots & -A^{T} & 0 & \cdots & 0 \\
0 & \cdots & 0 & 0 & \cdots & 0 & 0 & \cdots & 0 \\
\vdots & & \vdots & \vdots & & \vdots & \vdots & \ddots & \vdots \\
0 & \cdots & 0 & 0 & \cdots & 0 & 0 & \cdots & 0
\end{array}\right) \in \operatorname{sl}_{n_{i+1}}(\mathbb{C})
$$

где $A \in \mathrm{sl}_{n_{i}}(\mathbb{C})$, а $A^{T}$ - это матрица, являющаяся транспонированной матрицей к матрице $A$, то такие алгебры являются диагональными типа $A$.

Для таких алгебр доказано, что группа внешних автоморфизмов есть либо $\mathbb{Z}_{2} \times A(\mathrm{~g})$, либо $A(\mathrm{~g})$, где $\mathbb{Z}_{2}$ - это группа второго порядка, а $A(\mathrm{~g})$ - это не более чем счетная свободная абелева группа. Таким образом, для диагональной алгебры Ли типа $A$ определен естественный инвариант, являющийся рангом группы $A(\mathrm{~g})$. Показано, что для любого значения этого инварианта существует соответствуюшая диагональная алгебра Ли типа $A$.

Опишем структуру статьи. Во втором параграфе аккуратно определяется группа внешних автоморфизмов не более чем счетномерных локально простых алгебр Ли. Приведено доказательство теоремы 2.1 и утверждения 2.6, которые являются основным техническим средством для вычисления группы внешних автоморфизмов. Третий параграф перекликается с работой [4]. В нем дается более формальное описание категории диагональных алгебр Ли типа $A$. В четвертом параграфе доказывается теорема 4.1, в которой возникает группа $A(\mathrm{~g})$ и приводится серия примеров для которых вычисляется группа $A(\mathrm{~g})$.

\section{§2. Группы внешних автоморфизмов локально простых алгебр Ли}

Пусть $\mathfrak{C}$ - это произвольная категория. Определим категорию $\widehat{\mathfrak{C}}$ в два шага. Шаг 1. Определим категорию $\widetilde{\mathfrak{C}}$.

а) Объекты: объект $X$ категории $\widetilde{\mathfrak{C}}$ есть пара последовательностей

$$
\left(\left\{X_{i} \mid X_{i} \in \mathrm{ObC}\right\}_{i=1}^{\infty},\left\{x_{i} \mid x_{i} \in \operatorname{Hom}_{\mathfrak{C}}\left(X_{i}, X_{i+1}\right)\right\}_{i=1}^{\infty}\right)
$$


Неформально говоря, объект $X$ - это индуктивная система:

$$
X_{1} \stackrel{x_{1}}{\longrightarrow} X_{2} \stackrel{x_{2}}{\longrightarrow} X_{3} \rightarrow \cdots \rightarrow X_{i} \stackrel{x_{i}}{\longrightarrow} X_{i+1} \rightarrow \cdots
$$

Если $i \leqslant j$, то будем обозначать через $x_{i j}$ композицию $x_{j-1} \circ x_{j-2} \circ \cdots \circ x_{i}$ $\in \operatorname{Hom}_{\mathfrak{C}}\left(X_{i}, X_{j}\right)$. (Имеется в виду, что $x_{i i}=\operatorname{id}_{X_{i}}$.)

б) Морфизмы: если $X=\left(\left\{X_{i}\right\}_{i=1}^{\infty},\left\{x_{i}\right\}_{i=1}^{\infty}\right), Y=\left(\left\{Y_{i}\right\}_{i=1}^{\infty},\left\{y_{i}\right\}_{i=1}^{\infty}\right)$ суть объекты категории $\widetilde{\mathfrak{C}}$, то $\operatorname{Hom}_{\widetilde{\mathfrak{C}}}(X, Y)$ - это множество последовательностей $\left\{f_{i} \mid f_{i} \in\right.$ $\left.\operatorname{Hom}_{\mathfrak{C}}\left(X_{i}, Y_{n_{i}}\right)\right\}_{i=1}^{\infty}$ таких, что

$$
n_{1} \leqslant n_{2} \leqslant n_{3} \leqslant \cdots \leqslant n_{i} \leqslant n_{i+1} \leqslant \cdots
$$

и следующая диаграмма

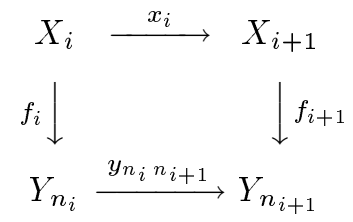

коммутативна для любого $i \geqslant 1$.

ПримеР. Пусть $X=\left(\left\{X_{i}\right\}_{i=1}^{\infty},\left\{x_{i}\right\}_{i=1}^{\infty}\right)$ есть объект категории $\widetilde{\mathfrak{C}}$, а $\left\{n_{i}\right\}_{i=1}^{\infty}-$ это возрастающая последовательность натуральных чисел такая, что $n_{i} \geqslant i$ для любого $i \geqslant 1$. Тогда последовательность $\left\{f_{i} \mid f_{i}=x_{i n_{i}} \in \operatorname{Hom}_{\mathfrak{C}}\left(X_{i}, X_{n_{i}}\right)\right\}_{i=1}^{\infty}$ задает морфизм в категории $\widetilde{\mathfrak{C}}$ из объекта $X$ в самого себя. Такие морфизмы будем называть тривиальнымми.

в) Композиция морфизмов: пусть

$$
X=\left(\left\{X_{i}\right\}_{i=1}^{\infty},\left\{x_{i}\right\}_{i=1}^{\infty}\right), \quad Y=\left(\left\{Y_{i}\right\}_{i=1}^{\infty},\left\{y_{i}\right\}_{i=1}^{\infty}\right), \quad Z=\left(\left\{Z_{i}\right\}_{i=1}^{\infty},\left\{z_{i}\right\}_{i=1}^{\infty}\right)
$$

- это объекты категории $\widetilde{\mathfrak{C}}_{\text {и }}$

$$
\begin{aligned}
& f=\left\{f_{i} \mid f_{i} \in \operatorname{Hom}_{\mathfrak{C}}\left(X_{i}, Y_{n_{i}}\right)\right\}_{i=1}^{\infty}, \\
& g=\left\{g_{i} \mid g_{i} \in \operatorname{Hom}_{\mathfrak{C}}\left(Y_{i}, Z_{m_{i}}\right)\right\}_{i=1}^{\infty}
\end{aligned}
$$

- это морфизмы категории $\widetilde{\mathfrak{C}}$.

Тогда положим

$$
g \circ f=\left\{(g \circ f)_{i} \mid(g \circ f)_{i}=g_{n_{i}} \circ f_{i} \in \operatorname{Hom}_{\mathfrak{C}}\left(X_{i}, Z_{m_{n_{i}}}\right)\right\}_{i=1}^{\infty}
$$

Например, композиция двух тривиальных морфизмов есть тривиальный морфизм. 
УТВеРЖДЕНИЕ 2.1. Данные а), б), в) задают категорию.

Шаг 2. Введем отношение $\sim$ на Моr $\widetilde{\mathfrak{C}}: f \sim f^{\prime}$ тогда и только тогда, когда $f, f^{\prime} \in \operatorname{Hom}_{\widetilde{\mathfrak{C}}}(X, Y)$ и существуют тривиальные морфизмы $t, t^{\prime} \in \operatorname{Hom}_{\widetilde{\mathfrak{C}}}(Y, Y)$ такие, что

$$
t \circ f=t^{\prime} \circ f^{\prime} .
$$

Непосредственной проверкой можно доказать следующее утверждение.

УТВЕРЖДЕНИЕ 2.2. а) Отношение есть отношение әквивалентности.

б) Отношение $\sim$ согласовано с композицией морфизмов. Это означает, что если $f, f^{\prime} \in \operatorname{Hom}_{\widetilde{\mathfrak{C}}}(X, Y)$ u $g, g^{\prime} \in \operatorname{Hom}_{\widetilde{\mathfrak{C}}}(Y, Z)$ maкие, ито $f \sim f^{\prime} u g \sim g^{\prime}$, mогда $g \circ f \sim g^{\prime} \circ f^{\prime}$.

Это утверждение позволяет определить категорию $\widehat{\mathfrak{C}}=\widetilde{\mathfrak{C}} /(\sim)$.

Неформально говоря, категория $\widehat{\mathfrak{C}}$ является фактором категории $\widetilde{\mathfrak{C}}$ по тривиальньм морфизмам.

Определим естественный функтор $\sigma: \mathfrak{C} \rightarrow \widehat{\mathfrak{C}}$.

a) На объектах: если $X \in \mathrm{Ob} \mathfrak{C}$, то тогда

$$
\sigma(X)=\left(\left\{X_{i} \mid X_{i}=X\right\}_{i=1}^{\infty},\left\{x_{i} \mid x_{i}=\operatorname{id}_{X}\right\}_{i=1}^{\infty}\right) .
$$

б) На морфизмах: если $f \in \operatorname{Hom}_{\mathfrak{C}}(X, Y)$, то тогда

$$
\sigma(f)=\left[\left\{f_{i} \mid f_{i}=f \in \operatorname{Hom}_{\mathfrak{C}}\left(X_{i}, Y_{i}\right)\right\}_{i=1}^{\infty}\right] .
$$

Здесь в квадратных скобках обозначен морфизм в категории $\widehat{\mathfrak{C}}$, который задается последовательностью $\left\{f_{i}\right\}_{i=1}^{\infty}$.

УТВЕРЖДЕНИЕ 2.3. Функтор $\sigma$ есть строгое и полное вложение категорий. Это означает, что следующее отображсение

$$
\sigma: \operatorname{Hom}_{\mathfrak{C}}(X, Y) \rightarrow \operatorname{Hom}_{\widehat{\mathcal{C}}}(\sigma(X), \sigma(Y))
$$

является биекцией для любых $X, Y \in \mathrm{ObC}$.

Пусть $\mathfrak{C}_{1}$ и $\mathfrak{C}_{2}$ суть произвольные категории и пусть задан функтор $F: \mathfrak{C}_{1} \rightarrow \mathfrak{C}_{2}$. Определим функтор $\widetilde{F}: \widetilde{\mathfrak{C}}_{1} \rightarrow \widetilde{\mathfrak{C}}_{2}$.

a) На объектах:

$$
\widetilde{F}\left(\left\{X_{i}\right\}_{i=1}^{\infty},\left\{x_{i}\right\}_{i=1}^{\infty}\right)=\left(\left\{F X_{i}\right\}_{i=1}^{\infty},\left\{F x_{i}\right\}_{i=1}^{\infty}\right)
$$

б) На морфизмах:

$$
\widetilde{F}\left\{f_{i}\right\}_{i=1}^{\infty}=\left\{F f_{i}\right\}_{i=1}^{\infty} .
$$

Ясно, что функтор $\widetilde{F}$ согласован с отношением эквивалентности $\sim$. Следовательно, определен функтор $\widehat{F}: \widehat{\mathfrak{C}}_{1} \rightarrow \widehat{\mathfrak{C}}_{2}$. 
ЗАмЕчАниЕ 1. Категория $\widehat{\mathfrak{C}}$ обладает тем свойством, что она содержит категорию $\mathfrak{C}$ в качестве полной подкатегории и, во-первых, любая индуктивная система вида:

$$
X_{1} \stackrel{x_{1}}{\longrightarrow} X_{2} \stackrel{x_{2}}{\longrightarrow} X_{3} \rightarrow \cdots \rightarrow X_{i} \stackrel{x_{i}}{\longrightarrow} X_{i+1} \rightarrow \cdots
$$

имеет прямой предел, лежаший в категории $\widehat{\mathfrak{C}}$, во-вторых, любой объект из категории $\widehat{\mathfrak{C}}$ является прямьм пределом индуктивной системы указанного вида. То есть, в некотором смысле, категория $\widehat{\mathfrak{C}}$ является пополнением категории $\mathfrak{C}$. Однако, в общем случае, такое пополнение не единственно, т.е. могут существовать не эквивалентные категории, обладаюшие указанным свойством и, скорее всего, среди них нет универсальной. Выбор именно такого пополнения обуславливается утверждением 2.4 .

Пусть $\mathfrak{C}_{1}$ - это категория конечномерных простых алгебр Ли. Обозначим через $\mathfrak{A}$ категорию не более чем счетномерных локально простых алгебр Ли.

УТВЕРЖДЕНИЕ 2.4. Категории $\mathfrak{A}$ и $\widehat{\mathfrak{C}}_{1}$ әквивалентны.

ДокАЗАТЕЛЬСтво. Для доказательства построим функтор $L: \widehat{\mathfrak{C}}_{1} \rightarrow \mathfrak{A}$ такой, что

1) $L$ - это строгий и полный функтор.

2) Если $Y$ - это объект категории $\mathfrak{A}$, то тогда существует объект $X \in \mathrm{Ob} \widehat{\mathfrak{C}}_{1}$ такой, что объект $L(X)$ изоморфен объекту $Y$.

Известно [5], что если функтор удовлетворяет этим двум свойствам, то он задает эквивалентность категорий.

Сначала построим функтор $\mathbf{L}: \widetilde{\mathfrak{C}}_{1} \rightarrow \mathfrak{A}$.

a) На объектах: пусть $X \in \mathrm{Ob} \widetilde{\mathfrak{C}}_{1}$ есть пара последовательностей $\left(\left\{\mathrm{g}_{i}\right\}_{i=1}^{\infty}\right.$, $\left.\left\{\varphi_{i}\right\}_{i=1}^{\infty}\right)$, где $\mathrm{g}_{i}-$ это конечномерная простая алгебра Ли и $\varphi_{i} \in \operatorname{Hom}_{\mathfrak{A}}\left(\mathrm{g}_{i}, \mathrm{~g}_{i+1}\right)-$ это гомоморфное вложение для любого $i \geqslant 1$. Тогда положим $\mathbf{L}(X)=\mathrm{g}=\lim _{\boldsymbol{~}} \mathrm{g}_{i}$.

б) На морфизмах: пусть последовательность

$$
f=\left\{f_{i} \mid f_{i} \in \operatorname{Hom}_{\mathfrak{C}_{1}}\left(\mathrm{~g}_{i}, \mathrm{~g}_{n_{i}}^{\prime}\right)\right\}_{i=1}^{\infty}
$$

задает морфизм в категории $\widetilde{\mathfrak{C}}$ из объекта $X=\left(\left\{\mathrm{g}_{i}\right\}_{i=1}^{\infty},\left\{\varphi_{i}\right\}_{i=1}^{\infty}\right)$ в объект $X^{\prime}=\left(\left\{\mathrm{g}_{i}^{\prime}\right\}_{i=1}^{\infty},\left\{\varphi_{i}^{\prime}\right\}_{i=1}^{\infty}\right)$. Определим поточечно гомоморфизм $\mathbf{L}(f)$ из алгебры Ли $\mathrm{g}=\lim _{\mathrm{g}} \mathrm{g}_{i}$ в алгебру Ли $\mathrm{g}^{\prime}=\lim _{i}^{\prime} \mathrm{g}_{i}^{\prime}$ Если $g \in \mathrm{g}$, то тогда существует $j \geqslant 1$ такой, что $\overrightarrow{g \in \mathrm{g}_{j}}$. Положим $\mathbf{L}(f)(g)=f_{j}(g) \in \mathrm{g}_{n_{j}}^{\prime} \subset \mathrm{g}^{\prime}$.

Во-первых, это определение корректно. То есть, если выбрать другой индекс $k$ такой, что $g \in \mathrm{g}_{k}$, то $f_{j}(g)$ совпадает с $f_{k}(g)$ как элемент прямого предела $\lim _{i} \mathrm{~g}_{i}^{\prime}=\mathrm{g}^{\prime}$.

Во-вторых, $\mathbf{L}(f)$ является гомоморфизмом алгебр Ли, так как каждый $f_{i}$ есть гомоморфизм алгебр Ли.

Рассмотрим отображение $\mathbf{L}: \operatorname{Hom}_{\tilde{\mathfrak{C}}}\left(X, X^{\prime}\right) \rightarrow \operatorname{Hom}_{\mathfrak{A}}\left(\mathrm{g}, \mathrm{g}^{\prime}\right)$ и перечислим его свойства. 
1. Это отображсение сюрвективно. Пусть $f \in \operatorname{Hom}_{\mathfrak{A}}\left(\mathrm{g}, \mathrm{g}^{\prime}\right)$, тогда для любого $i \geqslant 1$, существует $n_{i}$ так, что $f\left(\mathrm{~g}_{i}\right) \subset \mathrm{g}_{n_{i}}^{\prime}$. Тем самым определена последовательность $\left\{f_{i} \mid f_{i} \in \operatorname{Hom}_{\mathfrak{C}_{1}}\left(\mathrm{~g}_{i}, \mathrm{~g}_{n_{i}}^{\prime}\right)\right\}_{i=1}^{\infty}$, которая очевидным образом задает морфизм в категории $\widetilde{\mathfrak{C}}_{1}$.

2. Если последовательности $f=\left\{f_{i} \mid f_{i} \in \operatorname{Hom}_{\mathfrak{C}_{1}}\left(\mathrm{~g}_{i}, \mathrm{~g}_{n_{i}}^{\prime}\right)\right\}_{i=1}^{\infty} u f^{\prime}=$ $\left\{f_{i}^{\prime} \mid f_{i}^{\prime} \in \operatorname{Hom}_{\mathfrak{C}_{1}}\left(\mathrm{~g}_{i}, \mathrm{~g}_{n_{i}^{\prime}}^{\prime}\right)\right\}_{i=1}^{\infty}$ задают әквивалентные в категории $\widetilde{\mathfrak{C}}_{1}$ мор-

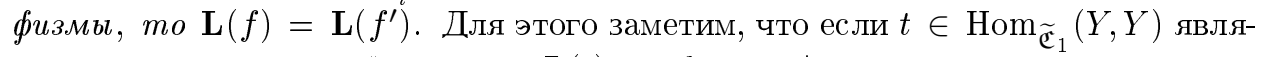
ется тривиальным морфизмом, то $\mathbf{L}(t)=\mathrm{id}_{\mathbf{L}(Y)}$. А теперь вспомним, что эквивалентность морфизмов $f, f^{\prime}$ означает существование тривиальных морфизмов и $t, t^{\prime} \in \operatorname{Hom}_{\widetilde{\mathfrak{C}}_{1}}\left(X^{\prime}, X^{\prime}\right)$ таких, что $t \circ f=t^{\prime} \circ f^{\prime}$. Следовательно, $\mathbf{L}(f)=\mathbf{L}\left(f^{\prime}\right)$.

3. Если морфизми $f=\left\{f_{i} \mid f_{i} \in \operatorname{Hom}_{\mathfrak{C}_{1}}\left(\mathrm{~g}_{i}, \mathrm{~g}_{n_{i}}^{\prime}\right)\right\}_{i=1}^{\infty} u f^{\prime}=\left\{f_{i}^{\prime} \mid f_{i}^{\prime} \in\right.$ $\left.\operatorname{Hom}_{\mathfrak{C}_{1}}\left(\mathrm{~g}_{i}, \mathrm{~g}_{n_{i}^{\prime}}^{\prime}\right)\right\}_{i=1}^{\infty}$ таковьи, что $\mathbf{L}(f)=\mathbf{L}\left(f^{\prime}\right)$, то $f$ и $f^{\prime}$ суть әквивалентнье морфизмы. В самом деле, если гомоморфизмы $\mathbf{L}(f), \mathbf{L}\left(f^{\prime}\right)$ из алгебры Ли g в алгебру Ли $\mathrm{g}^{\prime}$ совпадают, то совпадают и их ограничения на подалгебры Ли $\mathrm{g}_{i}$ для любого $i \geqslant 1$. Пусть $m_{i}$ - это такое число, что $\mathbf{L}(f)\left(\mathrm{g}_{i}\right), \mathbf{L}\left(f^{\prime}\right)\left(\mathrm{g}_{i}\right) \subset \mathrm{g}_{m_{i}}^{\prime}$ и $m_{i} \geqslant n_{i}, n_{i}^{\prime}$. Тогда

$$
\varphi_{n_{i} m_{i}} \circ f_{i}=\varphi_{n_{i}^{\prime} m_{i}} \circ f_{i}^{\prime}
$$

А это и означает, что морфизмы $f$ и $f^{\prime}$ эквивалентны.

Свойство 2 позволяет определить отображение

$$
L: \operatorname{Hom}_{\widetilde{\mathfrak{C}}_{1}}\left(X, X^{\prime}\right) /(\sim) \rightarrow \operatorname{Hom}_{\mathfrak{A}}\left(\mathrm{g}, \mathrm{g}^{\prime}\right)
$$

и, следовательно, функтор $L: \widehat{\mathfrak{C}}_{1} \rightarrow \mathfrak{A}$. Из свойств 1 и 3 следует, что этот функтор является строгим и полным. То, что функтор $L$ удовлетворяет второму условию следует из того факта, что любая счетномерная локально простая алгебра Ли является прямым пределом индуктивной системы следующего вида

$$
\mathrm{g}_{1} \stackrel{\varphi_{1}}{\longrightarrow} \mathrm{g}_{2} \stackrel{\varphi_{2}}{\longrightarrow} \mathrm{g}_{3} \rightarrow \cdots \rightarrow \mathrm{g}_{i} \stackrel{\varphi_{i}}{\longrightarrow} \mathrm{g}_{i+1} \rightarrow \cdots
$$

где $\mathrm{g}_{i}$ - это простая конечномерная алгебра Ли для любого $i \geqslant 1$. Следовательно, категории $\widehat{\mathfrak{C}}_{1}$ и $\mathfrak{A}$ эквивалентны.

Свяжем с каждой конечномерной простой алгеброй Ли $\mathrm{g} \in \mathrm{Ob}_{1}$ связную односвязную группу Ли $G$ такую, что $\operatorname{Lie}(G)=\mathrm{g}$. Как известно [2], сушествует гомоморфизм групп Ли $\mathrm{Ad}: G \rightarrow \operatorname{Aut}(\mathrm{g})$, причем образом этого отображения является нормальная подгруппа Ли, которая называется группой внутренних автомор$\oint$ фзмов алгебры Ли g и обозначается через Int $(\mathrm{g})$. Факторгруппа Aut(g)/ Int(g) называется группой внешних автоморфизмов алгебры Ли g.

Определим отношение $\sim$ на Mor $\mathfrak{C}_{1}: \varphi \sim \varphi^{\prime}$ тогда и только тогда, когда $\varphi, \varphi^{\prime} \in$ $\operatorname{Hom}_{\mathfrak{C}_{1}}\left(\mathrm{~g}_{1}, \mathrm{~g}_{2}\right)$ и сушествует элемент $g \in G_{2}$ такой, что $\varphi=\operatorname{Ad}(g) \circ \varphi^{\prime}$. Из того, что отображение Ad является гомоморфизмом групп, следует, что отношение есть отношение эквивалентности. 
Отображение Аd удовлетворяет следуюшему свойству. Пусть $\varphi \in \operatorname{Hom}_{\mathfrak{C}_{1}}\left(\mathrm{~g}_{1}, \mathrm{~g}_{2}\right)$ - это гомоморфизм алгебр Ли и пусть

$$
f_{\varphi}: G_{1} \rightarrow G_{2}
$$

такой гомоморфизм групп Ли, что $d f_{\varphi}=\varphi$. Тогда

$$
\varphi \circ \operatorname{Ad}(g)=\operatorname{Ad}\left(f_{\varphi}(g)\right) \circ \varphi
$$

для любого $g \in G_{1}$.

Из этого свойства следует, что отношение эквивалентности $\sim$ согласованно с композицией морфизмов в категории $\mathfrak{C}_{1}$. Следовательно, определена категория $\mathfrak{C}_{2}=\mathfrak{C}_{1} /(\sim)$ и функтор проекции $F: \mathfrak{C}_{1} \rightarrow \mathfrak{C}_{2}$.

Неформально говоря, категория $\mathfrak{C}_{2}-$ это категория конечномерных простых алгебр Ли, в которой морфизмы рассматриваются с точностью до внутренних сопряжений.

Из определения следует, что группа внешних автоморфизмов алгебры Ли $\mathrm{g} \in \mathrm{ObC}_{1}$ есть $\operatorname{Aut}_{\mathfrak{C}_{2}}(F(\mathrm{~g}))$.

Пусть алгебра Ли $\mathrm{g} \in \mathrm{Ob} \mathfrak{A}$. Рассмотрим ее как объект категории $\widehat{\mathfrak{C}}_{1}$, т.е. представим ее в виде индуктивной системы:

$$
\mathrm{g}_{1} \stackrel{\varphi_{1}}{\longrightarrow} \mathrm{g}_{2} \stackrel{\varphi_{2}}{\longrightarrow} \mathrm{g}_{3} \rightarrow \cdots \rightarrow \mathrm{g}_{i} \stackrel{\varphi_{i}}{\longrightarrow} \mathrm{g}_{i+1} \rightarrow \cdots,
$$

где $\mathrm{g}_{i} \in \mathrm{Ob}_{1}$.

Тогда существует индуктивная система

$$
G_{1} \stackrel{f_{12}}{\longrightarrow} G_{2} \stackrel{f_{23}}{\longrightarrow} G_{3} \rightarrow \cdots \rightarrow G_{i} \stackrel{f_{i(i+1)}}{\longrightarrow} G_{i+1} \rightarrow \cdots
$$

где $G_{i}$ - это связная односвязная группа Ли такая, что $\operatorname{Lie}\left(G_{i}\right)=\mathrm{g}_{i}$ и $d f_{i(i+1)}=\varphi_{i}$ для любого $i \geqslant 1$.

Пусть $G=\underline{\lim } G_{i}$ и $f_{i}: G_{i} \rightarrow G$ - это соответствуюшие отображения.

Пусть задана система $\mathfrak{V}$ подмножеств группы $G$, содержаших единицу, которая обладает следующими свойствами.

а) Пересечение двух множеств системы $\mathfrak{V}$ содержит некоторое третье множество системы $\mathfrak{V}$.

б) Если множество $U$ принадлежит семейству $\mathfrak{V}$, то существует $V \in \mathfrak{V}$ так, что $V V^{-1} \subset U$.

в) Если множество $U$ принадлежит семейству $\mathfrak{V}$ и $a$ является элементом множества $U$, то существует $V \in \mathfrak{V}$ так, что $V a \subset U$.

г) Если множество $U$ принадлежит семейству $\mathfrak{V}$ и $a$ является элементом группы $G$, то сушествует $V \in \mathfrak{V}$ так, что $a^{-1} V a \subset U$.

Тогда известно [6], что семейство множеств вида $U g$, где $U \in \mathfrak{V}$ и $g \in G$, является базой некоторой топологии, относительно которой группа $G$ преврашается в топологическую группу и семейство $\mathfrak{V}$ является базой окрестностей единицы.

Пусть $\mathfrak{U}=\left\{C\left(f_{i}\left(G_{i}\right)\right)\right\}_{i=1}^{\infty}$, где $C\left(f_{i}\left(G_{i}\right)\right)$ - это централизатор подгруппы $f_{i}\left(G_{i}\right)$ в группе $G$. 
УТВЕРЖДЕНИЕ 2.5. Семейство централизаторов $\mathfrak{U}$ обладает всеми въшеуказанными свойствами.

Из этого утверждения следует, что $G$ - это топологическая группа и, следовательно, можно определить пополнение $\widehat{G}$ группы $G$ по соответствующей топологии.

Определим гомоморфизм Ad : $\widehat{G} \rightarrow \operatorname{Aut}_{\mathfrak{A}}(\mathrm{g})$, где g - это $\lim _{i} g_{i}$

Сначала построим гомоморфизм Ad : $G \rightarrow \operatorname{Aut}_{\mathfrak{A}}(\mathrm{g})$.

Пусть $g \in G$, тогда существуют $j \geqslant 1$ и $g_{j} \in G_{j}$ такие, что $f_{j}\left(g_{j}\right)=g$. Элемент $g_{j}$ определяет автоморфизм $\operatorname{Ad}\left(g_{j}\right)$ алгебры Ли $g_{j}$. Элемент $f_{j(j+k)}\left(g_{j}\right)$ определяет автоморфизм $\operatorname{Ad}\left(f_{j(j+k)}\left(g_{j}\right)\right)$ алгебры Ли $g_{j+k}$ такой, что

$$
\varphi_{j+k-1} \circ \operatorname{Ad}\left(f_{j(j+k-1)}\left(g_{j}\right)\right)=\operatorname{Ad}\left(f_{j(j+k)}\left(g_{j}\right)\right) \circ \varphi_{j+k-1}
$$

для любого $k \geqslant 1$. Следовательно, определена последовательность

$$
\begin{aligned}
\left\{h_{i} \mid h_{i}=\operatorname{Ad}\left(f_{j i}\left(g_{j}\right)\right) \in \operatorname{Hom}_{\mathfrak{C}_{1}}\left(\mathrm{~g}_{i}, \mathrm{~g}_{i}\right) \text { при } i\right. & \geqslant j \text { и } \\
h_{i}=\left.\operatorname{Ad}\left(g_{j}\right)\right|_{\mathrm{g}_{i}} & \left.\in \operatorname{Hom}_{\mathfrak{C}_{1}}\left(\mathrm{~g}_{i}, \mathrm{~g}_{j}\right) \text { при } i<j\right\}_{i=1}^{\infty},
\end{aligned}
$$

которая задает автоморфизм в категории $\widehat{\mathfrak{C}}$ и, следовательно, автоморфизм $\operatorname{Ad}(g)$ алгебры Ли g. Нетрудно убедиться в том, что эта конструкция корректна.

Напомним, что элементами пополнения $\widehat{G}$ являются последовательности $\left\{g_{i} \mid\right.$ $\left.g_{i} \in G\right\}_{i=1}^{\infty}$ такие, что

$$
\forall k \exists N \text { такой, что } \forall s, t \geqslant N g_{s}^{-1} g_{t} \in C\left(f_{k}\left(G_{k}\right)\right) \text {. }
$$

Если $g=\left\{g_{i} \mid g_{i} \in G\right\}_{i=1}^{\infty} \in \widehat{G}$, то определена последовательность $\left\{\operatorname{Ad}\left(g_{i}\right)\right\}_{i=1}^{\infty}$ автоморфизмов алгебры Ли g. Эта последовательность автоморфизмов поточечно сходится к некоторому автоморфизму. В самом деле, если $x \in \mathrm{g}$, то тогда, используя условие $(*)$, получаем, что последовательность $\left\{\operatorname{Ad}\left(g_{i}\right)(x)\right\}_{i=1}^{\infty}$ элементов алгебры Ли g стабилизируется на некотором элементе $\operatorname{Ad}(g)(x)$. Следовательно, мы определили гомоморфизм групп $\mathrm{Ad}: \widehat{G} \rightarrow \operatorname{Aut}_{\mathfrak{A}}(\mathrm{g})$.

ЗАмечАниЕ 2. Морфизм, задаваемый последовательностью

$$
f=\left\{f_{i} \mid f_{i} \in \operatorname{Hom}_{\mathfrak{C}_{1}}\left(\mathrm{~g}_{i}, \mathrm{~g}_{n_{i}}\right)\right\}_{i=1}^{\infty} \in \operatorname{Hom}_{\widehat{\mathfrak{C}}}(\mathrm{g}, \mathrm{g})
$$

принадлежит образу $\operatorname{Ad}(\widehat{G})$ тогда и только тогда, когда сушествует последовательность $\left\{g_{i} \mid g_{i} \in G_{n_{i}}\right\}_{i=1}^{\infty}$ такая, что гомоморфизм алгебр Ли $f_{i}: g_{i} \rightarrow g_{n_{i}}$ есть ограничение автоморфизма $\operatorname{Ad}\left(g_{i}\right)$ на подалгебру Ли $g_{i}$. Ясно, что $\operatorname{Ad}(\widehat{G})$ является нормальной подгруппой в группе $\operatorname{Aut}_{\mathfrak{A}}(\mathrm{g})$. Обозначим $\operatorname{Ad}(\widehat{G})$ через $\operatorname{Int}(\mathrm{g})$.

ОПРЕДЕЛЕНИЕ 2.1. Группой внешних автоморфизмов алгебры Ли $g \in \mathfrak{A}$ будем назьвать факторгруппу $\operatorname{Aut}_{\mathfrak{A}}(\mathrm{g}) / \operatorname{Int}(\mathrm{g})$. 
TeOpema 2.1. Группа внешних автоморфизмов алгебры Ли $\mathrm{g} \in \mathrm{Ob} \mathfrak{A}$ изоморфна группе $\operatorname{Aut}_{\widehat{\mathfrak{C}}_{2}}(\widehat{F}(\mathrm{~g}))$, где $\operatorname{Aut}_{\widehat{\mathfrak{C}}_{2}}(\widehat{F}(\mathrm{~g}))$ - это группа обратимых әлементов в полугруппе $\operatorname{Hom}_{\widehat{\mathfrak{C}}_{2}}(\widehat{F}(\mathrm{~g}), \widehat{F}(\mathrm{~g}))$.

ДокАЗАтЕльСтво. Рассмотрим алгебру Ли g как объект категории $\widehat{\mathfrak{C}}_{1}$, т.е. g - это индуктивная система

$$
\mathrm{g}_{1} \stackrel{\varphi_{1}}{\longrightarrow} \mathrm{g}_{2} \stackrel{\varphi_{2}}{\longrightarrow} \mathrm{g}_{3} \rightarrow \cdots \rightarrow \mathrm{g}_{i} \stackrel{\varphi_{i}}{\longrightarrow} \mathrm{g}_{i+1} \rightarrow \cdots
$$

где $\mathrm{g}_{i} \in \mathrm{Ob}_{1} \mathfrak{C}_{1}$

В любом случае, существует отображение

$$
\widehat{F}: \operatorname{Hom}_{\widehat{\mathfrak{C}}_{1}}(\mathrm{~g}, \mathrm{~g}) \rightarrow \operatorname{Hom}_{\widehat{\mathfrak{C}}_{2}}(\widehat{F}(\mathrm{~g}), \widehat{F}(\mathrm{~g})),
$$

являюшееся гомоморфизмом полугрупп и, следовательно, переводящее обратимые элементы полугрупшы Hom $_{\widehat{\mathfrak{C}}_{1}}(\mathrm{~g}, \mathrm{~g})$ в обратимые элементы полугрупшы $\operatorname{Hom}_{\widehat{\mathfrak{C}}_{2}}(\widehat{F}(\mathrm{~g}), \widehat{F}(\mathrm{~g}))$.

Воспользуемся следующей несложной леммой.

Лемма 2.1. Пусть $S_{1}$ и $S_{2}$ суть полугруппьи с единицей. Пусть $S_{1}^{*}$ и $S_{2}^{*}$ суть группь обратимых әлементов полугрупп $S_{1}$ u $S_{2}$. Пусть $\sigma: S_{1} \rightarrow S_{2}$ - это сюрвективный гомоморфизм полугрупп такой, что если $\sigma(s)=1$, то $s \in S_{1}^{*}$. Тогда ограничение гомоморфизма $\sigma$ на группу $S_{1}^{*}$ является сюрвективныцм гомоморфизмом на группу $S_{2}^{*}$.

Докажем, что отображение $\widehat{F}$ является сюръективным. Пусть задан морфизм

$$
x=\left[\left\{x_{i} \mid x_{i} \in \operatorname{Hom}_{\mathfrak{C}_{2}}\left(F\left(\mathrm{~g}_{i}\right), F\left(\mathrm{~g}_{n_{i}}\right)\right)\right\}_{i=1}^{\infty}\right] \in \operatorname{Hom}_{\widehat{\mathfrak{C}}_{2}}(\widehat{F}(\mathrm{~g}), \widehat{F}(\mathrm{~g}))
$$

в категории $\widehat{\mathfrak{C}}_{2}$. Тогда по определению

$$
F\left(\varphi_{n_{i} n_{i+1}}\right) \circ x_{i}=x_{i+1} \circ F\left(\varphi_{i}\right)
$$

для любого $i \geqslant 1$.

Пусть $\left\{h_{i}^{\prime} \mid h_{i}^{\prime} \in \operatorname{Hom}_{\mathfrak{C}_{1}}\left(\mathrm{~g}_{i}, \mathrm{~g}_{n_{i}}\right)\right\}_{i=1}^{\infty}-$ это такая последовательность, что $F\left(h_{i}^{\prime}\right)=x_{i}$ для любого $i \geqslant 1$. Тогда сушествует $g_{i+1} \in G_{n_{i+1}}$ такой, что

$$
\varphi_{n_{i} n_{i+1}} \circ h_{i}^{\prime}=\operatorname{Ad}\left(g_{i+1}\right) \circ h_{i+1}^{\prime} \circ \varphi_{i}
$$

для любого $i \geqslant 1$.

Положим

$$
\begin{aligned}
& h_{1}=h_{1}^{\prime}, \\
& h_{2}=\operatorname{Ad}\left(g_{2}\right) \circ h_{2}^{\prime} \text {, } \\
& h_{3}=\operatorname{Ad}\left(f_{n_{2} n_{3}}\left(g_{2}\right) g_{3}\right) \circ h_{3}^{\prime} \text {, }
\end{aligned}
$$

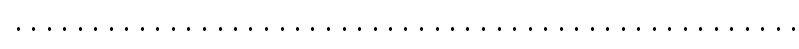

$$
\begin{aligned}
& h_{i}=\operatorname{Ad}\left(f_{n_{2} n_{i}}\left(g_{2}\right) f_{n_{3} n_{i}}\left(g_{3}\right) \ldots f_{n_{i-1} n_{i}}\left(g_{i-1}\right) g_{i}\right) \circ h_{i}^{\prime},
\end{aligned}
$$

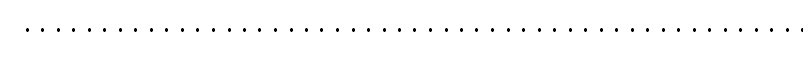


Тогда непосредственными выкладками можно показать, что последовательность $\left\{h_{i}\right\}_{i=1}^{\infty}$ определяет морфизм в категории $\widehat{\mathfrak{C}}_{1}$, т.е. вьполнено равенство

$$
\varphi_{n_{i} n_{i+1}} \circ h_{i}=h_{i+1} \circ \varphi_{i}
$$

для любого $i \geqslant 1$. Так как гомоморфизмы $h_{i}$ и $h_{i}^{\prime}$ сопряжены, то $\widehat{F}(h)=x$.

Пусть последовательность $h=\left\{h_{i}\right\}_{i=1}^{\infty} \in \operatorname{Hom}_{\widehat{\mathfrak{C}}_{1}}(\mathrm{~g}, \mathrm{~g})$ задает такой морфизм, что $\widehat{F}(h)=\operatorname{id}_{\widehat{F}(\mathrm{~g})}$. Тогда $F\left(h_{i}\right)=F\left(\varphi_{n_{i}-1}\right) \circ \cdots \circ F\left(\varphi_{i+1}\right) \circ F\left(\varphi_{i}\right)$. Это равенство означает, что для любого $i \geqslant 1$, сушествует элемент $g_{i} \in G_{n_{i}}$ такой, что $h_{i}=$ $\operatorname{Ad}\left(g_{i}\right) \circ \varphi_{n_{i}-1} \circ \cdots \circ \varphi_{i+1} \circ \varphi_{i}$. По замечанию 2 получаем, что $h \in \operatorname{Int}(\mathrm{g})$.

Пусть $h \in \operatorname{Int}(\mathrm{g})$. Из замечания 2 получаем, что существует последовательность $\left\{g_{i} \mid g_{i} \in G_{n_{i}}\right\}_{i=1}^{\infty}$ такая, что

$$
h_{i}=\operatorname{Ad}\left(g_{i}\right) \circ \varphi_{n_{i}-1} \circ \cdots \circ \varphi_{i+1} \circ \varphi_{i} .
$$

Следовательно, $F\left(h_{i}\right)=F\left(\varphi_{n_{i}-1}\right) \circ \cdots \circ F\left(\varphi_{i+1}\right) \circ F\left(\varphi_{i}\right)$. А значит, $\widehat{F}(h)=\mathrm{id}_{\widehat{F}(\mathrm{~g})}$.

Из леммы 2.1 получаем, что гомоморфизм групा

$$
\widehat{F}: \operatorname{Aut}_{\widehat{\mathfrak{C}}_{1}}(\mathrm{~g}) \rightarrow \operatorname{Aut}_{\widehat{\mathfrak{C}}_{2}}(\widehat{F}(\mathrm{~g}))
$$

сюръективен и $\operatorname{Ker} \widehat{F}=\operatorname{Int}(\mathrm{g})$.

Следуюшее утверждение будет часто использоваться в дальнейшем.

УТВЕРЖДЕНИЕ 2.6. Пусть $\mathfrak{C}-$ произвольная категория $и \mathfrak{C}^{\prime} \subset \mathfrak{C}$ такая ее подкатегория, что для морфизмов категории $\mathfrak{C}^{\prime}$ выполнено следующее: если $f \in \operatorname{Hom}_{\mathfrak{C}^{\prime}}(X, Y)$ u $f=g \circ h$, əде $h \in \operatorname{Hom}_{\mathfrak{C}}(X, Z)$ u $g \in \operatorname{Hom}_{\mathfrak{C}}(Z, Y)$, mo $h \in \operatorname{Hom}_{\mathfrak{C}^{\prime}}(X, Z)$. Тогда $\operatorname{Aut}_{\widehat{\mathfrak{C}}}(X)=\operatorname{Aut}_{\widehat{\mathfrak{C}}^{\prime}}(X)$ для любого $X \in \mathrm{Ob} \widehat{\mathfrak{C}}^{\prime}$.

ДокаЗАТЕльство. Если $X=\left(\left\{X_{i}\right\}_{i=1}^{\infty},\left\{x_{i}\right\}_{i=1}^{\infty}\right) \in \mathrm{Ob} \widehat{\mathfrak{C}}^{\prime}$ и

$$
\left\{f_{i} \mid f_{i} \in \operatorname{Hom}_{\mathfrak{C}}\left(X_{i}, X_{n_{i}}\right)\right\}_{i=1}^{\infty} \in \operatorname{Aut}_{\widehat{\mathfrak{C}}}(X)
$$

тогда сушествует последовательность

$$
\left\{h_{i} \mid h_{i} \in \operatorname{Hom}_{\mathfrak{C}}\left(X_{i}, X_{m_{i}}\right)\right\}_{i=1}^{\infty} \in \operatorname{Aut}_{\widehat{\mathfrak{C}}}(X)
$$

такая, что

$$
h_{n_{i}} \circ f_{i}=x_{m_{n_{i}}-1} \circ \cdots \circ x_{i} .
$$

Так как $x_{m_{n_{i}}-1} \circ \cdots \circ x_{i} \in \operatorname{Mor} \widehat{\mathfrak{C}^{\prime}}$, то $f_{i} \in \operatorname{Mor} \widehat{\mathfrak{C}}^{\prime}$. 


\section{§ 3. Диагональные алгебры Ли типа $A$ и полугруппа $\mathbf{D}$}

Локально простую алгебру Ли g будем называть алгеброй Ли типа $A$, если она является прямым пределом индуктивной системы следующего вида

$$
\operatorname{sl}_{n_{1}}(\mathbb{C}) \stackrel{\varphi_{1}}{\longrightarrow} \operatorname{sl}_{n_{2}}(\mathbb{C}) \stackrel{\varphi_{2}}{\longrightarrow} \operatorname{sl}_{n_{3}}(\mathbb{C}) \longrightarrow \cdots \longrightarrow \operatorname{sl}_{n_{i}}(\mathbb{C}) \stackrel{\varphi_{i}}{\longrightarrow} \operatorname{sl}_{n_{i+1}}(\mathbb{C}) \longrightarrow \cdots
$$

Выберем для каждой алгебры Ли $\mathrm{sl}_{n}(\mathbb{C})$ представление минимальной размерности, обозначим его через $V_{n}$ и назовем его тавтологическим. Тогда $\operatorname{dim} V_{n}=n$ и $_{n}(\mathbb{C})=\operatorname{sl}\left(V_{n}\right)$, т.е. это множество линейных операторов на линейном пространстве $V_{n}$ с нулевым следом. Будем называть двойственное представление $V_{n}^{*}$ aнтuтавтологическим и одномерное представление $\mathbb{C}$ тривиальнылм.

Если алгебра Ли $\mathrm{sl}_{k}(\mathbb{C})$ вкладьвается в алгебру Ли $\mathrm{sl}_{n}(\mathbb{C})$, то любое представление алгебры $Л_{и} \operatorname{sl}_{n}(\mathbb{C})$ является представлением алгебры $Л_{\text {и }} \mathrm{sl}_{k}(\mathbb{C})$, в том числе и тавтологическое представление $V_{n}$ алгебры Ли $\operatorname{sl}_{n}(\mathbb{C})$ является некоторым представлением алгебры Ли $\mathrm{sl}_{k}(\mathbb{C})$.

ОПРЕДЕЛЕНИЕ 3.1 . Вложение $\varphi: \mathrm{sl}_{k}(\mathbb{C}) \rightarrow \operatorname{sl}_{n}(\mathbb{C})$ будем называть диагональным. если представление $V_{n}$ есть сумма тавтологических, антитавтологических и тривиальных представлений алгебры Ли $\mathrm{sl}_{k}(\mathbb{C})$.

Ясно, что композиция диагональных вложений является диагональным вложением. То есть можно определить подкатегорию $\mathfrak{D}_{1}$ в категории конечномерных простых алгебр Ли $\mathfrak{C}_{1}$.

а) Объекты подкатегории $\mathfrak{D}_{1}$ - это алгебры Ли $\operatorname{sl}_{n}(\mathbb{C})$.

б) Морфизмы подкатегории $\mathfrak{D}_{1}$ - это диагональные вложения.

Объекты категории $\widehat{\mathfrak{D}}_{1}$ будем называть диагональными алгебрами Ли ти$n a A$, т.е. диагональная алгебра Ли g - это локально простая алгебра Ли, являюшаяся прямым пределом индуктивной системы следующего вида

$$
\operatorname{sl}_{n_{1}}(\mathbb{C}) \stackrel{\varphi_{1}}{\longrightarrow} \operatorname{sl}_{n_{2}}(\mathbb{C}) \stackrel{\varphi_{2}}{\longrightarrow} \operatorname{sl}_{n_{3}}(\mathbb{C}) \longrightarrow \cdots \longrightarrow \operatorname{sl}_{n_{i}}(\mathbb{C}) \stackrel{\varphi_{i}}{\longrightarrow} \operatorname{sl}_{n_{i+1}}(\mathbb{C}) \longrightarrow \cdots
$$

где вложения $\varphi_{i}: \operatorname{sl}_{n_{i}}(\mathbb{C}) \rightarrow \operatorname{sl}_{n_{i+1}}(\mathbb{C})$ являются диагональными для любого $i \geqslant 1$.

Для диагональных вложений верен следующий факт [3]. Пусть коммутативная диаграмма

$$
\begin{gathered}
\operatorname{sl}_{k}(\mathbb{C}) \stackrel{\varphi}{\longrightarrow} \operatorname{sl}_{n}(\mathbb{C}) \\
\psi \searrow \underset{ }{\nearrow} \operatorname{sl}_{m}(\mathbb{C})
\end{gathered}
$$

такова, ито вложение $\varphi$ является диагональным. Тогда вложение $\psi$ также является диагональным. Следовательно, для подкатегории $\mathfrak{D}_{1}$ вьполнено условие утверждения 2.6. Значит, если g - это диагональная алгебра Ли, то

$$
\operatorname{Aut}_{\widehat{\mathfrak{C}}_{1}}(\mathrm{~g})=\operatorname{Aut}_{\widehat{\mathfrak{D}}_{1}}(\mathrm{~g}) .
$$


Также определена подкатегория $\mathfrak{D}_{2}$ в категории $\mathfrak{C}_{2}$.

а) Объекты подкатегории $\mathfrak{D}_{2}$ - это алгебры Ли $\operatorname{sl}_{n}(\mathbb{C})$, где $n \geqslant 3$.

б) Морфизмы подкатегории $\mathfrak{D}_{2}$ - это диагональные вложения с точностью до сопряжения.

Требование $n \geqslant 3$ объясняется тем, что тавтологическое и антитавтологическое представления алгебры Ли $\mathrm{sl}_{2}(\mathbb{C})$ изоморфны.

$\mathrm{K}$ подкатегории $\mathfrak{D}_{2}$ можно также применить утверждение 2.6. Следовательно, если $\mathrm{g} \in \mathrm{Ob} \widehat{\mathfrak{D}}_{1}$, то тогда группа внешних автоморфизмов алгебры Ли g изоморфна группе

$$
\operatorname{Aut}_{\widehat{\mathfrak{D}}_{2}}(\widehat{F}(\mathrm{~g})) \text {. }
$$

Зафиксируем некоторые обозначения. Если $A_{1}, A_{2}, \ldots, A_{s}$ - это числовые матрицы размеров $k_{1} \times k_{1}, k_{2} \times k_{2}, \ldots, k_{s} \times k_{s}$ соответственно, то через $\operatorname{diag}\left(A_{1}, A_{2}, \ldots, A_{s}\right)$ обозначим числовую матрицу размера $\left(k_{1}+k_{2}+\cdots+k_{s}\right) \times$ $\left(k_{1}+k_{2}+\cdots+k_{s}\right)$ следуюшего вида:

$$
\left(\begin{array}{cccc}
A_{1} & 0 & \cdots & 0 \\
0 & A_{2} & \cdots & 0 \\
\cdots & \cdots & \cdots & \cdots \\
0 & 0 & \cdots & A_{s}
\end{array}\right)
$$

Опишем более формально категорию $\mathfrak{D}_{2}$. Для этого заметим, что с точностью до сопряжения диагональное вложение $\varphi: \operatorname{sl}_{k}(\mathbb{C}) \rightarrow \operatorname{sl}_{n}(\mathbb{C})$, где $k \geqslant 3$, задается тройкой неотрицательных целых чисел $(a, b, c)$, где $a, b, c-$ это, соответственно, кратности тавтологических, антитавтологических и тривиальных подпредставлений алгебры Ли $\mathrm{sl}_{k}(\mathbb{C})$ в тавтологическом представлении $V_{n}$ алгебры Ли $\mathrm{sl}_{n}(\mathbb{C})$.

Если заданы два диагональных вложения $\psi: \operatorname{sl}_{k}(\mathbb{C}) \rightarrow \operatorname{sl}_{m}(\mathbb{C})$ и $\chi: \operatorname{sl}_{m}(\mathbb{C}) \rightarrow$ $\operatorname{sl}_{n}(\mathbb{C})$ так, что вложению $\psi$ соответствует тройка $(a, b, c)$, а вложению $\chi$ соответствует тройка $(x, y, z)$, то вложению $\chi \circ \psi$ соответствует тройка

$$
(a x+b y, a y+b x,(x+y) c+z) \text {. }
$$

Это следует из того, что можно выбрать базисы в пространствах $V_{k}, V_{m}, V_{n}$ так, что $\psi(A)=\operatorname{diag}(\underbrace{A, \ldots, A}_{a}, \underbrace{-A^{T}, \ldots,-A^{T}}_{b}, \underbrace{0, \ldots, 0}_{c})$, где $A \in \mathrm{sl}_{k}(\mathbb{C})$ и $\chi(B)=\operatorname{diag}(\underbrace{B, \ldots, B}_{x}, \underbrace{-B^{T}, \ldots,-B^{T}}_{y}, \underbrace{0, \ldots, 0}_{z})$, где $B \in \operatorname{sl}_{m}(\mathbb{C})$.

Тогда число экземпляров матрицы $A$ в матрице $\chi(\psi(A))$, т.е. кратность тавтологического представления $V_{k}$ в представлении $V_{n}$, равно $a x+b y$, число матриц $-A^{T}$, стояших на диагонали матрицы $\chi(\psi(A))$, равно $a y+b x$, а число нулей на диагонали матрицы $\chi(\psi(A))$ равно $(x+y) c+z$.

Из этого следует, что можно определить полугруппу D. Элементами полугруппы $\mathbf{D}$ являются тройки неотрицательных целых чисел $(a, b, c)$, где хотя бы одно из чисел $a$ или $b$ не равно нулю. Умножение таких троек задается по формуле

$$
(a, b, c) *(x, y, z)=(a x+b y, a y+b x,(x+y) c+z) .
$$


Полугруппа $\mathbf{D}$ действует справа на множестве натуральных чисел больших либо равных трем $\mathbb{N}_{\geqslant 3}$ следующим образом

$$
n *(a, b, c)=n(a+b)+c .
$$

Это действие происходит из того соображения, что если диагональному вложению $\varphi: \mathrm{sl}_{k}(\mathbb{C}) \rightarrow \operatorname{sl}_{n}(\mathbb{C})$ соответствует тройка $(a, b, c) \in \mathbf{D}$, то тогда $n=k *(a, b, c)$.

Эти соображения позволяют описать категорию $\mathfrak{D}_{2}$ следуюшим образом.

а) Объекты категории $\mathfrak{D}_{2}$ - это натуральные числа большие либо равные трем.

б) Морфизмы категории $\mathfrak{D}_{2}:$ если $k, n \in \mathbb{N}_{\geqslant 3}$, то $\operatorname{Hom}_{\mathfrak{D}_{2}}(k, n)=\{d \in \mathbf{D} \mid$ $k * d=n\}$.

Опишем строение полугрупшы D. Пусть $S$ - это полугруппа пар $(a, b)$ неотрицательных целых чисел, где хотя бы одно из чисел $a$ или $b$ не равно нулю, и умножение таких пар происходит по формуле

$$
(x, y) *(a, b)=(a x+b y, a y+b x) .
$$

Полугруппа $\mathbf{D}$ содержит полугруппу $S$ в качестве подполугруппы таких троек $(a, b, c)$, что $c=0$.

Непосредственными вычислениями можно доказать следующее утверждение.

УТВеРЖДЕНИЕ 3.1. а) Полугруппа $S$ коммутативна.

б) Отображение $\chi: S \rightarrow \mathbb{N}, \chi((a, b))=a+b$, является гомоморфизмом из полугруппь $S$ в полугруппу натуральных чисел $\mathbb{N}$ по умножению.

в) Отображение $\kappa: S \rightarrow \mathbb{Z}, \kappa((a, b))=(a-b)$, является гомоморфизмом из полугруппы $S$ в полугруппу $\mathbb{Z}$ чельх чисел по умножению.

Полугруппа $\mathbf{D}$ содержит полугруппу неотрицательных целых чисел по сложению $\mathbb{Z}_{\geqslant 0}$ в качестве подполугруппы троек вида $(1,0, c)$.

УТВЕРЖДЕНИЕ 3.2. Полугруппа $\mathbf{D}$ изоморфна полупрямому произведению полугрупп $S \ltimes \mathbb{Z}_{\geqslant 0}$ с законом умножения

$$
\left(g_{1}, c_{1}\right) *\left(g_{2}, c_{2}\right)=\left(g_{1} * g_{2}, \chi\left(g_{2}\right) c_{1}+c_{2}\right) .
$$

ДокАЗАТЕЛЬСтво. Это следует из определения полугрупा $\mathbf{D}$ и $S$.

Представим полугруппу $S$ в виде объединения двух своих подмножеств $S=$ $S_{\infty} \cup S_{0}$, где $S_{\infty}$ - это множество пар $(a, b)$ таких, что $a \neq b$, а $S_{0}$ - это множество пар $(a, b)$ таких, что $a=b$.

УТВЕРЖДЕНИЕ 3.3. а) Подмножество $S_{\infty}$ является подполугруппой, причем если $g_{1}, g_{2} \in S$ u $g_{1} * g_{2} \in S_{\infty}$, то $g_{1}, g_{2} \in S_{\infty}$.

б) Подмножество $S_{0}$ является идеалом в полугруппе $S$. То есть $g_{1} * g_{2} \in$ $S_{0}$ для любъх $g_{1} \in S$ u $g_{2} \in S_{0}$. 
ДокАЗАТЕЛЬство. а) Подмножество $S_{\infty}$ есть множество таких элементов $g \in S$, что $\kappa(g) \neq 0$. Отсюда следует, что если $\kappa\left(g_{1} * g_{2}\right) \neq 0$, то $\kappa\left(g_{1}\right) \neq 0$ и $\kappa\left(g_{2}\right) \neq 0$, так как $\kappa$-это гомоморфизм.

б) Подмножество $S_{0}$ есть множество таких элементов $g \in S$, что $\kappa(g)=0$. Отсюда следует, что если $g_{2} \in S_{0}$, то $\kappa\left(g_{1} * g_{2}\right)=0$ для любого $g_{1} \in S$.

Напомним определение полугруппы с сокращением.

ОПРЕДЕЛЕНИЕ 3.2. Коммутативную полугруппу $P$ будем назьвать сократимой, если из равенства $x * y=z * y$ следует равенство $x=z$ для любых $x, y, z \in P$.

Следующее утверждение было доказано в работе [4].

УТВЕРЖ ДЕНИЕ 3.4. а) Полугруппа $S_{\infty}$ сократима.

б) Полугруппа $S_{0}$ сократима.

Пусть $\mathbf{D}_{\infty}$ - это подмножество троек $(a, b, c)$ полугрупшы $\mathbf{D}$ таких, что $(a, b) \in S_{\infty}$. И пусть $\mathbf{D}_{0}$ - это подмножество троек $(a, b, c)$ полугруппы $\mathbf{D}$ таких, что $(a, b) \in S_{0}$. Из утверждений 3.2 и 3.3 следует, что $\mathbf{D}_{0}$ является идеалом в полугрупше $\mathbf{D}$ и $\mathbf{D}_{\infty}$ является такой подполугруппой в полугруппе $\mathbf{D}$, что если $d_{1}, d_{2} \in \mathbf{D}$ и $d_{1} * d_{2} \in \mathbf{D}_{\infty}$, то тогда $d_{1}, d_{2} \in \mathbf{D}_{\infty}$.

Определим подкатегорию $\mathfrak{D}_{\infty}$ в категории $\mathfrak{D}_{2}$.

a) Объекты категории $\mathfrak{D}_{\infty}$ такие же как в категории $\mathfrak{D}_{2}$. То есть это натуральные числа большие либо равные трем.

б) Морфизмы категории $\mathfrak{D}_{\infty}$ : если $k, n \in \mathbb{N}_{\geqslant 3}$, то $\operatorname{Hom}_{\mathfrak{D}}(k, n)=\left\{d \in \mathbf{D}_{\infty} \mid\right.$ $d * k=n\}$.

Определим также подкатегорию $\mathfrak{D}_{0}$ в категории $\mathfrak{D}_{2}$.

a) Объекты: $\mathrm{Ob} \mathfrak{D}_{0}=\mathrm{Ob} \mathfrak{D}_{2}=\mathbb{N}_{\geqslant 3}$.

б) Морфизмы категории $\mathfrak{D}_{0}:$ если $k, n \in \mathbb{N}_{\geqslant 3}$ и $k \neq n$, то $\operatorname{Hom}_{\mathfrak{D}_{0}}(k, n)=$ $\left\{d \in \mathbf{D}_{0} \mid d * k=n\right\}$. Если $k=n$, то $\operatorname{Hom}_{\mathfrak{D}_{0}}(k, k)$ - это множество, состоящее из одного тождественного морфизма $\mathrm{id}_{k}$.

Tеорема 3.1. Если $X \in \mathrm{Ob} \widehat{\mathfrak{D}}_{2}$, то тогда возможны два варианта:

а) существует $Y \in \widehat{\mathfrak{D}}_{\infty}$ такой, что

$$
\operatorname{Aut}_{\widehat{\mathfrak{D}}_{2}}(X)=\operatorname{Aut}_{\widehat{\mathfrak{D}}_{\infty}}(Y) ;
$$

б) существует $Y \in \widehat{\mathfrak{D}}_{0}$ такой, что

$$
\operatorname{Aut}_{\widehat{\mathfrak{D}}_{2}}(X)=\operatorname{Aut}_{\widehat{\mathfrak{D}}_{0}}(Y) .
$$


ДОКАЗАТЕЛЬСТво. Пусть $X$ - это пара последовательностей

$$
\left(\left\{n_{i} \mid n_{i} \in \mathbb{N}_{\geqslant 3}\right\}_{i=1}^{\infty},\left\{x_{i} \mid x_{i} \in \mathbf{D}, n_{i} * x_{i}=n_{i+1}\right\}_{i=1}^{\infty}\right)
$$

Тогда множество натуральных чисел $\mathbb{N}$ есть объединение своих подмножеств $\mathbb{N}_{0}$ и $\mathbb{N}_{\infty}$, где

$$
\begin{aligned}
\mathbb{N}_{0} & =\left\{k \in \mathbb{N} \mid x_{k} \in \mathbf{D}_{0}\right\}, \\
\mathbb{N}_{\infty} & =\left\{k \in \mathbb{N} \mid x_{k} \in \mathbf{D}_{\infty}\right\} .
\end{aligned}
$$

Подмножество $\mathbb{N}_{0}$ может быть конечным или бесконечным.

a) Предположим, что множество $\mathbb{N}_{0}$ конечно. Рассмотрим $Y=\left(\left\{n_{i}\right\}_{i=m+1}^{\infty}\right.$, $\left.\left\{x_{i}\right\}_{i=m+1}^{\infty}\right)$, где $m=\max _{k \in \mathbb{N}_{0}} k$. Так как $x_{i} \in \mathbf{D}_{\infty}$ для любого $i>m$, то мы можем рассматривать $Y$ и как объект категории $\widehat{\mathfrak{D}}_{2}$, и как объект категории $\widehat{\mathfrak{D}}_{\infty}$.

Ясно, что $Y$ изоморфен объекту $X$ как объект категории $\widehat{\mathfrak{D}}_{2}$. Следовательно,

$$
\operatorname{Aut}_{\widehat{\mathfrak{D}}_{2}}(X)=\operatorname{Aut}_{\widehat{\mathfrak{D}}_{2}}(Y) .
$$

Из утверждений 3.3 и 2.6 следует, что

$$
\operatorname{Aut}_{\widehat{\mathfrak{D}}_{2}}(Y)=\operatorname{Aut}_{\widehat{\mathfrak{D}}_{\infty}}(Y) \text {. }
$$

б) Предположим, что множество $\mathbb{N}_{0}$ бесконечно. Тогда существует строго возрастаюшая последовательность натуральных чисел $\left\{i_{k}\right\}_{k=1}^{\infty}$ такая, что $x_{i_{k}} \in \mathbf{D}_{0}$. Рассмотрим $Y=\left(\left\{l_{k} \mid l_{k}=n_{i_{k}}\right\}_{k=1}^{\infty},\left\{y_{k} \mid y_{k}=x_{i_{k} i_{k+1}}\right\}_{k=1}^{\infty}\right)$. Так как $\mathbf{D}_{0}$ является идеалом полугрупшы $\mathbf{D}$, то $x_{i_{k} i_{k+1}} \in \mathbf{D}_{0}$. Следовательно, можно рассматривать $Y$ как объект категории $\widehat{\mathfrak{D}}_{2}$ или как объект категории $\widehat{\mathfrak{D}}_{0}$.

Ясно, что объект $X$ изоморфен объекту $Y$ в категории $\widehat{\mathfrak{D}}_{2}$. Следовательно,

$$
\operatorname{Aut}_{\widehat{\mathfrak{D}}_{2}}(X)=\operatorname{Aut}_{\widehat{\mathfrak{D}}_{2}}(Y) \text {. }
$$

Для завершения доказательства надо показать, что

$$
\operatorname{Aut}_{\widehat{\mathfrak{D}}_{2}}(Y)=\operatorname{Aut}_{\widehat{\mathfrak{D}}_{0}}(Y)
$$

В самом деле, если последовательность

$$
f=\left\{f_{i} \mid f_{i} \in \operatorname{Hom}_{\mathfrak{D}_{2}}\left(l_{i}, l_{r_{i}}\right)\right\}_{i=1}^{\infty}
$$

задает автоморфизм объекта $Y$ в категории $\widehat{\mathfrak{D}}_{2}$, то тогда последовательность

$$
h=\left\{h_{i} \mid h_{i}=y_{r_{i}} \circ f_{i} \in \operatorname{Hom}_{\mathfrak{D}_{2}}\left(l_{i}, l_{r_{i}+1}\right)\right\}_{i=1}^{\infty}
$$

задает тот же самый автоморфизм объекта $Y$ в категории $\widehat{\mathfrak{D}}_{2}$, но, на этот раз, $h_{i} \in \mathbf{D}_{0}$ для любого $i \geqslant 1$, так как $\mathbf{D}_{0}-$ это идеал в полугруппе $\mathbf{D}$. 


\section{§4. Группы внешних автоморфизмов диагональных алгебр Ли}

Пусть $P$ - это коммутативная сократимая полугруппа. Напомним определение группы частных $Q(P)$.

ОПРЕДЕЛЕНИЕ 4.1. Пусть $P \times P$ - это декартов квадрат полугрупшы $P$. Пусть $\sim$ - это отношение эквивалентности на $P \times P$ такое, что $(a, s) \sim\left(a^{\prime}, s^{\prime}\right)$ тогда и только тогда, когда $a * s^{\prime}=a^{\prime} * s$. Положим $Q(P)=P \times P /(\sim)$.

Элемент $(a, s) \in Q(P)$ обычно обозначается через $a / s$.

ПримеР. Пусть $\mathbb{N}$ - это полугруппа натуральных чисел по умножению. Тогда $Q(\mathbb{N})=\mathbb{Q}_{>0}$, где $\mathbb{Q}_{>0}-$ это группа положительных рациональных чисел по умножению.

Пусть $P$ - это произвольная полугруппа. Определим категорию $\mathfrak{D}_{P}$.

a) Объекты: категория $\mathfrak{D}_{P}$ содержит только один объект. Обозначим этот объект точкой •

б) Морфизмы: $\operatorname{Hom}_{\mathfrak{D}_{P}}(\cdot, \cdot)=P_{e}$, где $P_{e}-$ это полугруппа $P$, если в полугруппе $P$ сушествует единища, или $P_{e}=P \cup\{e\}$, где $e$ - это присоединенная единица, в том случае, если в полугруппе $P$ нет единицы.

В соответствии с конструкцией из $\S 2$, можно рассмотреть категорию $\widehat{\mathfrak{D}}_{P}$.

УТВеРЖДЕНИЕ 4.1. Пусть $P$ - это коммутативная сократимая полугруппа. Тогда полугруппа $\operatorname{Hom}_{\widehat{\mathfrak{D}}_{P}}(X, X)$ естественно вкладывается в группу $Q(P)$ для любого обвекта $X \in \mathrm{Ob} \widehat{\mathfrak{D}}_{P}$.

ДокАЗАТЕЛЬСтво. Опишем категорию $\widetilde{\mathfrak{D}}_{P}$ в более удобном виде. Так как в категории $\mathfrak{D}_{P}$ всего один объект, то можно считать, что объектами категории $\widetilde{\mathfrak{D}}_{P}$ являются последовательности $\left\{p_{i} \mid p_{i} \in P\right\}_{i=1}^{\infty}$. Морфизмами из объекта $\left\{p_{i} \mid p_{i} \in P\right\}_{i=1}^{\infty}$ в объект $\left\{p_{i}^{\prime} \mid p_{i}^{\prime} \in P\right\}_{i=1}^{\infty}$ являются пары последовательностей $\left(\left\{f_{i} \mid f_{i} \in P\right\}_{i=1}^{\infty},\left\{k_{i} \mid k_{i} \in \mathbb{N}\right\}_{i=1}^{\infty}\right)$ такие, что последовательность $\left\{k_{i}\right\}_{i=1}^{\infty}$ является возрастающей последовательностью натуральных чисел и $p_{k_{i} k_{i+1}}^{\prime} * f_{i}=$ $f_{i+1} * p_{i}$ для любого $i \geqslant 1$. (Напомним, что если $s \geqslant t$, то $p_{s t}^{\prime}=p_{s-1}^{\prime} * p_{s-2}^{\prime} * \cdots * p_{t}^{\prime}$.)

Определим отображение

$$
\begin{gathered}
\rho: \operatorname{Hom}_{\tilde{\mathfrak{D}}_{P}}(X, X) \rightarrow Q(P), \\
\rho\left(\left\{f_{i}\right\}_{i=1}^{\infty},\left\{k_{i}\right\}_{i=1}^{\infty}\right)=\frac{f_{1}}{p_{1 k_{1}}}=\frac{f_{1}}{p_{1} * p_{2} * \cdots * p_{k_{1}-1}},
\end{gathered}
$$

где $X=\left\{p_{i} \mid p_{i} \in P\right\}_{i=1}^{\infty}$. Предположим, что два морфизма

$$
f=\left(\left\{f_{i}\right\}_{i=1}^{\infty},\left\{k_{i}\right\}_{i=1}^{\infty}\right)
$$

и

$$
f^{\prime}=\left(\left\{f_{i}^{\prime}\right\}_{i=1}^{\infty},\left\{k_{i}^{\prime}\right\}_{i=1}^{\infty}\right)
$$


совпадают как морфизмы категории $\widehat{\mathfrak{D}}_{P}$. В частности, это означает, что существует число $l \geqslant k_{1}, k_{1}^{\prime}$ такое, что

$$
f_{1} * p_{k_{1} l}=f_{1}^{\prime} * p_{k_{1}^{\prime} l} .
$$

Умножив это равенство на $p_{1 k_{1}} * p_{1 k_{1}^{\prime}}$, мы получим равенство

$$
f_{1} * p_{1 k_{1}^{\prime}} * p_{1 l}=f_{1}^{\prime} * p_{1 k_{1}} * p_{1 l} .
$$

Сокрашая полученное равенство на $p_{1 l}$, получаем равенство

$$
f_{1} * p_{1 k_{1}^{\prime}}=f_{1}^{\prime} * p_{1 k_{1}}
$$

которое означает, что

$$
\frac{f_{1}}{p_{1 k_{1}}}=\frac{f_{1}^{\prime}}{p_{1 k_{1}^{\prime}}} .
$$

Следовательно, можно определить отображение

$$
\widehat{\rho}: \operatorname{Hom}_{\widehat{\mathfrak{D}}_{P}}(X, X) \rightarrow Q(P) .
$$

Докажем инъективность отображения $\widehat{\rho}$. Пусть пары последовательностей $f=\left(\left\{f_{i}\right\}_{i=1}^{\infty},\left\{k_{i}\right\}_{i=1}^{\infty}\right), f^{\prime}=\left(\left\{f_{i}^{\prime}\right\}_{i=1}^{\infty},\left\{k_{i}^{\prime}\right\}_{i=1}^{\infty}\right)$ задают такие морфизмы в категории $\hat{\mathfrak{D}}_{P}$, что $\widehat{\rho}(f)=\widehat{\rho}\left(f^{\prime}\right)$. Тогда $f_{1} / p_{1 k_{1}}=f_{1}^{\prime} / p_{1 k_{1}^{\prime}}$ или, другими словами,

$$
f_{1} * p_{1 k_{1}^{\prime}}=f_{1}^{\prime} * p_{1 k_{1}}
$$

Из того, что $f, f^{\prime}$ являются морфизмами вытекают равенства

$$
\begin{aligned}
& p_{k_{1} k_{i}} * f_{1}=f_{i} * p_{1 i}, \\
& p_{k_{1}^{\prime} k_{i}^{\prime}} * f_{1}^{\prime}=f_{i}^{\prime} * p_{1 i}
\end{aligned}
$$

для любого $i \geqslant 1$.

Выберем число $l$ такое, что $l \geqslant k_{i}, k_{i}^{\prime}$. Тогда

$$
p_{1 l}=p_{1 k_{1}} * p_{k_{1} k_{i}} * p_{k_{i} l}=p_{1 k_{1}^{\prime}} * p_{k_{1}^{\prime} k_{i}^{\prime}} * p_{k_{i}^{\prime} l} .
$$

Следовательно, из равенства

$$
p_{1 l} * f_{1} * p_{1 k_{1}^{\prime}}=p_{1 l} * f_{1}^{\prime} * p_{1 k_{1}}
$$

вытекает равенство

$$
f_{i} * p_{1 i} * p_{1 k_{1}} * p_{k_{i} l} * p_{1 k_{1}^{\prime}}=f_{i}^{\prime} * p_{1 i} * p_{1 k_{1}^{\prime}} * p_{k_{i}^{\prime} l} * p_{1 k_{1}} .
$$

Сокрашая последнее равенство на $p_{1 i} * p_{1 k_{1}} * p_{1 k_{1}^{\prime}}$, получаем равенство

$$
f_{i} * p_{k_{i} l}=f_{i}^{\prime} * p_{k_{i}^{\prime} l}
$$

для любого $i \geqslant 1$. А это и означает, что последовательности $f$ и $f^{\prime}$ задают один и тот же морфизм в категории $\widehat{\mathfrak{D}}_{P}$.

Докажем, что $\widehat{\rho}$ является гомоморфизмом полугрупп. 
ЛЕмма 4.1. Если последовательность $f=\left\{f_{i} \mid f_{i} \in P\right\}_{i=1}^{\infty}$ задает морфизм обгекта $\left\{p_{i} \mid p_{i} \in P\right\}_{i=1}^{\infty}$ в категории $\widetilde{\mathfrak{D}}_{P}$, mо

$$
\frac{f_{1}}{p_{1 k_{1}}}=\frac{f_{m}}{p_{m k_{m}}}
$$

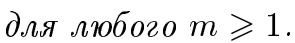

ДоКАЗАТЕЛЬСТво. Поскольку $f$ является морфизмом, то вьполнено равенстBO

$$
p_{k_{1} k_{m}} * f_{1}=f_{m} * p_{1 m}
$$

Умножив это равенство на $p_{m k_{m}}$, получим равенство

$$
p_{k_{1} k_{m}} * p_{m k_{m}} * f_{1}=f_{m} * p_{1 k_{m}} .
$$

Так как $p_{1 k_{m}}=p_{1 k_{1}} * p_{k_{1} k_{m}}$, то, сократив последнее равенство на $p_{k_{1} k_{m}}$, получим

$$
p_{m k_{m}} * f_{1}=f_{m} * p_{1 k_{1}} .
$$

То есть

$$
\frac{f_{1}}{p_{1 k_{1}}}=\frac{f_{m}}{p_{m k_{m}}}
$$

Пусть пары последовательностей

$$
f=\left(\left\{f_{i}\right\}_{i=1}^{\infty},\left\{k_{i}\right\}_{i=1}^{\infty}\right), \quad f^{\prime}=\left(\left\{f_{i}^{\prime}\right\}_{i=1}^{\infty},\left\{k_{i}^{\prime}\right\}_{i=1}^{\infty}\right)
$$

задают морфизмы объекта $\left\{p_{i} \mid p_{i} \in P\right\}_{i=1}^{\infty}$ в категории $\widehat{\mathfrak{D}}_{P}$. Тогда композиция $f^{\prime} \circ f$ задается парой последовательностей $\left(\left\{f_{k_{i}}^{\prime} * f_{i}\right\}_{i=1}^{\infty},\left\{k_{k_{i}}^{\prime}\right\}_{i=1}^{\infty}\right)$. Следовательно,

$$
\widehat{\rho}\left(f^{\prime} \circ f\right)=\frac{f_{k_{1}}^{\prime} * f_{1}}{p_{1 k_{k_{1}}}^{\prime}}=\frac{f_{k_{1}}}{p_{k_{1} k_{k_{1}}}} * \frac{f_{1}}{p_{1 k_{1}}}=\frac{f_{k_{1}}^{\prime}}{p_{k_{1} k_{k_{1}}}^{\prime}} * \widehat{\rho}(f)=\widehat{\rho}\left(f^{\prime}\right) * \widehat{\rho}(f) .
$$

Последнее равенство выполнено в силу леммы 4.1.

В предыдушем параграфе были определены полугруппы $S_{\infty}, S_{0}$ и категории $\widehat{\mathfrak{D}}_{\infty}, \widehat{\mathfrak{D}}_{0}$. Определим функтор $C_{\infty}: \widehat{\mathfrak{D}}_{\infty} \rightarrow \widehat{\mathfrak{D}}_{S_{\infty}}$.

a) На объектах: если $X \in \mathrm{Ob} \widehat{\mathfrak{D}}_{\infty}$, то тогда $X$ есть пара последовательностей $\left(\left\{n_{i}\right\}_{i=1}^{\infty},\left\{d_{i}\right\}_{i=1}^{\infty}\right)$, где $d_{i} \in \mathbf{D}_{\infty}$. Из утверждения 3.2 следует, что $d_{i}$ есть пара $\left(s_{i}, c_{i}\right)$, где $s_{i} \in S_{\infty}$. Тогда положим $C_{\infty}(X)=\left\{s_{i}\right\}_{i=1}^{\infty} \in \mathrm{Ob} \widehat{\mathfrak{D}}_{S_{\infty}}$.

б) На морфизмах: пусть заданы объекты категории $\widehat{\mathfrak{D}}_{\infty}$

$$
\begin{aligned}
X & =\left(\left\{n_{i}\right\}_{i=1}^{\infty},\left\{d_{i}\right\}_{i=1}^{\infty}\right), \\
X^{\prime} & =\left(\left\{n_{i}^{\prime}\right\}_{i=1}^{\infty},\left\{d_{i}^{\prime}\right\}_{i=1}^{\infty}\right) .
\end{aligned}
$$

Пусть задан морфизм из объекта $X$ в объект $X^{\prime}$

$$
\begin{aligned}
f= & \left\{f_{i} \mid f_{i} \in \operatorname{Hom}_{\mathfrak{D}_{\infty}}\left(n_{i}, n_{k_{i}}^{\prime}\right), f_{i}=\left(r_{i}, t_{i}\right) \in \mathbf{D}_{\infty}\right\}_{i=1}^{\infty} \in \operatorname{Hom}_{\widehat{\mathfrak{D}}_{\infty}}\left(X, X^{\prime}\right) . \\
& \text { Тогда положим } C_{\infty}(f)=\left(\left\{r_{i}\right\}_{i=1}^{\infty},\left\{k_{i}\right\}_{i=1}^{\infty}\right) \in \operatorname{Hom}_{\widehat{\mathfrak{D}}_{S_{\infty}}}\left(C_{\infty}(X), C_{\infty}\left(X^{\prime}\right)\right) .
\end{aligned}
$$

Из утверждения 3.2 следует, что $C_{\infty}$ является функтором.

Совершено аналогично можно определить функтор $C_{0}: \widehat{\mathfrak{D}}_{0} \rightarrow \widehat{\mathfrak{D}}_{S_{0}}$. 
УТВЕРЖДЕНИЕ 4.2. а) Функтор $C_{\infty}$ является строгим. Это означает, что следующее отобрахсение

$$
C_{\infty}: \operatorname{Hom}_{\widehat{\mathfrak{D}}_{\infty}}\left(X, X^{\prime}\right) \rightarrow \operatorname{Hom}_{\widehat{\mathfrak{D}}_{S_{\infty}}}\left(C_{\infty}(X), C_{\infty}\left(X^{\prime}\right)\right)
$$

инвективно для любъх $X, X^{\prime} \in \mathrm{Ob} \mathfrak{D}_{\infty}$.

б) Функтор $C_{0}$ является строгим.

ДокАЗАТЕЛЬСтво. Воспользуемся следующей леммой.

ЛЕмма 4.2. Пусть заданы әлементы $\mathbf{D} \quad d_{1}=\left(s_{1}, c_{1}\right), d_{2}=\left(s_{2}, c_{2}\right) \in \mathbf{D}=$ $S \ltimes \mathbb{Z}$ и число $n \in \mathbb{N}_{\geqslant 3}$ такие, что $s_{1}=s_{2} u n * d_{1}=n * d_{2}$. Тогда $c_{1}=c_{2}$, m.e. $d_{1}=d_{2}$.

Зафиксируем объекты $X$ и $X^{\prime}$ в категории $\mathfrak{D}_{\infty}$

$$
\begin{aligned}
X & =\left(\left\{n_{i}\right\}_{i=1}^{\infty},\left\{d_{i} \mid d_{i} \in D_{\infty}, n_{i+1}=n_{i} * d_{i}\right\}_{i=1}^{\infty}\right), \\
X^{\prime} & =\left(\left\{n_{i}^{\prime}\right\}_{i=1}^{\infty},\left\{d_{i}^{\prime} \mid d_{i} \in D_{\infty}, n_{i+1}^{\prime}=n_{i}^{\prime} * d_{i}^{\prime}\right\}_{i=1}^{\infty}\right)
\end{aligned}
$$

Пусть морфизмы $f, f^{\prime} \in \operatorname{Hom}_{\widehat{\mathfrak{D}}_{\infty}}\left(X, X^{\prime}\right)$ задаются последовательностями

$$
\begin{aligned}
& \left\{f_{i} \mid f_{i}=\left(r_{i}, b_{i}\right) \in D_{\infty}=S_{\infty} \ltimes \mathbb{Z} ; n_{k_{i}}=n_{i} * f_{i}\right\}_{i=1}^{\infty}, \\
& \left\{f_{i}^{\prime} \mid f_{i}^{\prime}=\left(r_{i}^{\prime}, b_{i}^{\prime}\right) \in D_{\infty}=S_{\infty} \ltimes \mathbb{Z} ; n_{k_{i}^{\prime}}=n_{i} * f_{i}^{\prime}\right\}_{i=1}^{\infty} .
\end{aligned}
$$

Предположим, что $C_{\infty}(f)=C_{\infty}\left(f^{\prime}\right)$. Тогда это означает, что для любого $i \geqslant 1$ существует $l_{i} \geqslant k_{i}, k_{i}^{\prime}$ такое, что морфизмы $f$ и $f^{\prime}$ задаются последовательностями

$$
\begin{aligned}
& \left\{g_{i} \mid g_{i}=\left(s_{i}, c_{i}\right) \in D_{\infty}=S_{\infty} \ltimes \mathbb{Z} ; n_{l_{i}}=n_{i} * g_{i}\right\}_{i=1}^{\infty}, \\
& \left\{g_{i}^{\prime} \mid g_{i}^{\prime}=\left(s_{i}, c_{i}^{\prime}\right) \in D_{\infty}=S_{\infty} \ltimes \mathbb{Z} ; n_{l_{i}}=n_{i} * g_{i}^{\prime}\right\}_{i=1}^{\infty}
\end{aligned}
$$

Из леммы 4.2 следует, что $g_{i}=g_{i}^{\prime}$ для любого $i \geqslant 1$. То есть $f=f^{\prime}$.

Строгость функтора $C_{0}$ доказывается аналогично.

УТВЕРЖДЕНИЕ 4.3. Имеем: а) $Q\left(S_{\infty}\right)=\mathbb{Z}_{2} \times \mathbb{Z}^{\infty}$, б) $Q\left(S_{0}\right)=\mathbb{Z}^{\infty}$.

Здесь $\mathbb{Z}_{2}$ - это группа второго порядка, а $\mathbb{Z}^{\infty}$ - это свободная абелева группа бесконечного ранга.

ДокАзАтЕльство. а) Напомним, что определены два гомоморфизма полугрупा:

$$
\begin{aligned}
& \chi: S_{\infty} \rightarrow \mathbb{N} ; \quad \chi((a, b))=a+b, \\
& \kappa: S_{\infty} \rightarrow \mathbb{Z}_{\neq 0} ; \quad \kappa((a, b))=(a-b),
\end{aligned}
$$

где $\mathbb{Z}_{\neq 0}$ - это полугруппа ненулевых целых чисел по умножению.

Ясно, что следуюший гомоморфизм полугрупп

$$
S_{\infty} \stackrel{(\chi, \kappa)}{\longrightarrow} \mathbb{N} \times \mathbb{Z}_{\neq 0}
$$


является инъективным. Следовательно, существует инъективный гомоморфизм групा

$$
Q\left(S_{\infty}\right) \rightarrow Q(\mathbb{N}) \times Q\left(\mathbb{Z}_{\neq 0}\right)=\mathbb{Q}_{>0} \times \mathbb{Q}^{*},
$$

где $\mathbb{Q}^{*}$ - это группа обратимых элементов поля $\mathbb{Q}$.

Группа $\mathbb{Q}_{>0}$ изоморфна группе $\mathbb{Z}^{\infty}$. В качестве свободных образующих можно взять, например, простые натуральные числа. Группа $\mathbb{Q}^{*}$ изоморфна групе $\mathbb{Z}_{2} \times \mathbb{Z}^{\infty}$. Следовательно, группа $\mathbb{Q}_{>0} \times \mathbb{Q}^{*}$ есть $\mathbb{Z}_{2} \times \mathbb{Z}^{\infty}$.

Полугруппа $S_{\infty}$ содержит элемент $s=(0,1)$ такой, что $s^{2}=e$, где $e$ - это единица полугруппы $S_{\infty}$. Следовательно, $Q\left(S_{\infty}\right)=\mathbb{Z}_{2} \times F$, где $F$ - это подгруппа свободной абелевой группы $\mathbb{Z}^{\infty}$, а значит, $F$ - это не более чем счетная свободная абелева группа. Полугруппа $S_{\infty}$ содержит полугруппу $\mathbb{N}$ в качестве подполугруппы пар $(a, b)$ таких, что $b=0$. Следовательно, $F=\mathbb{Z}^{\infty}$, так как $Q(\mathbb{N})=\mathbb{Z}^{\infty}$.

б) Полугруппа $S_{0}$ изоморфна полугруппе $\mathbb{N}_{\text {even }}$, где $\mathbb{N}_{\text {even }}$ - это полугруппа четных натуральных чисел по умножению. Изоморфизм устанавливается гомоморфизмом $\kappa$. Следовательно,

$$
Q\left(S_{0}\right)=Q\left(\mathbb{N}_{\text {even }}\right)=Q(\mathbb{N})=\mathbb{Q}_{>0}=\mathbb{Z}^{\infty}
$$

Комбинируя теорему 3.1 и утверждения 4.1-4.3, получаем следуюшую теорему.

ТеОрема 4.1. Пусть g - это диагональная алгебра Ли типа А. Тогда группа внешних автоморфизмов алгебры Ли g изоморфна группе $\mathbb{Z}_{2} \times A(\mathrm{~g})$ или группе $A(\mathrm{~g})$, где $A(\mathrm{~g})$ - это не более чем счетная свободная абелева гpynna.

Заметим, что из этой теоремы не следует, что группа $A(\mathrm{~g})$ может быть нетривиальной. Для того чтобы привести примеры, показывающие, что группа $A(\mathrm{~g})$ может быть произвольного ранга, определим полные подкатегории $\widehat{\mathfrak{D}}_{\infty}^{\prime}, \widehat{\mathfrak{D}}_{0}^{\prime}$ в категориях $\widehat{\mathfrak{D}}_{\infty}, \widehat{\mathfrak{D}}_{0}$ соответственно.

a) Объекты категории $\widehat{\mathfrak{D}}_{\infty}^{\prime}$ - это такие пары последовательностей

$$
\left(\left\{n_{i} \mid n_{i} \in \mathbb{N}\right\}_{i=1}^{\infty},\left\{d_{i} \mid d_{i}=\left(s_{i}, c_{i}\right) \in \mathbf{D}_{\infty}=S_{\infty} \ltimes \mathbb{Z}_{\geqslant 0} ; n_{i+1}=n_{i} * d_{i}\right\}_{i=1}^{\infty}\right),
$$

что ряд

$$
\sum_{i=1}^{\infty} \frac{c_{i}}{\chi\left(s_{1}\right) \chi\left(s_{2}\right) \cdots \chi\left(s_{i}\right)}
$$

расходится.

б) Морфизмы в категории $\widehat{\mathfrak{D}}_{\infty}^{\prime}$ такие же, как и в категории $\widehat{\mathfrak{D}}_{\infty}$.

Категория $\widehat{\mathfrak{D}}_{0}^{\prime}$ определяется аналогично.

УТВЕРЖДЕНИЕ 4.4. а) Ограничение функтора $C_{\infty}$ на подкатегорию $\widehat{\mathfrak{D}}_{\infty}^{\prime}$ устанавливает әквивалентность категорий $\widehat{\mathfrak{D}}_{\infty}^{\prime} u \widehat{\mathfrak{D}}_{S_{\infty}}$.

б) Ограничение функтора $C_{0}$ на подкатегорию $\widehat{\mathfrak{D}}_{0}^{\prime}$ устанавливает эквивалентность категорий $\widehat{\mathfrak{D}}_{0}^{\prime} u \widehat{\mathfrak{D}}_{S_{0}}$. 
ДоказАтеЛьство. Докажем пункт а).

В силу утверждения 4.2 достаточно показать, что отображение

$$
C_{\infty}: \operatorname{Hom}_{\widehat{\mathfrak{D}}_{\infty}^{\prime}}\left(X^{\prime}, X\right) \rightarrow \operatorname{Hom}_{\widehat{\mathfrak{D}}_{S \infty}}\left(C_{\infty}\left(X^{\prime}\right), C_{\infty}(X)\right)
$$

сюръективно для любых $X, X^{\prime} \in \mathrm{Ob} \widehat{\mathfrak{D}}_{\infty}^{\prime}$.

Лемма 4.3. Пусть заданьи числа $m, n \in \mathbb{N}_{\geqslant 3}$ и последовательность $\left\{d_{i} \mid\right.$ $\left.d_{i}=\left(s_{i}, c_{i}\right) \in D=S \times \mathbb{Z}_{\geqslant 0}\right\}_{i=1}^{\infty}$ такая, ито ряд

$$
\sum_{i=1}^{\infty} \frac{c_{i}}{\chi\left(s_{1}\right) \chi\left(s_{2}\right) \cdots \chi\left(s_{i}\right)}
$$

расходится. Тогда существует $k \in \mathbb{N}$ такое, ито $\forall l \geqslant k$

$$
m \chi\left(s_{1}\right) \chi\left(s_{2}\right) \cdots \chi\left(s_{l}\right) \leqslant n * d_{1} * d_{2} * \cdots * d_{l}
$$

ДоКАЗАТЕЛЬСтво. По определению действия полугруппы $\mathbf{D}$ на множестве $\mathbb{N}_{\geqslant 3}$ получаем

$$
\begin{array}{r}
n * d_{1} * d_{2} * \cdots * d_{j}=n \chi\left(s_{1}\right) \chi\left(s_{2}\right) \chi\left(c_{3}\right) \cdots \chi\left(s_{j}\right)+c_{1} \chi\left(s_{2}\right) \chi\left(s_{3}\right) \cdots \chi\left(s_{j}\right) \\
+c_{2} \chi\left(s_{3}\right) \cdots \chi\left(s_{j}\right)+\cdots+c_{j-1} \chi\left(s_{j}\right)+c_{j} .
\end{array}
$$

Требуется сравнить это число с числом $m \chi\left(s_{1}\right) \chi\left(s_{2}\right) \cdots \chi\left(s_{j}\right)$. Разделим оба числа на $\chi\left(s_{1}\right) \chi\left(s_{2}\right) \chi\left(c_{3}\right) \cdots \chi\left(s_{j}\right)$ и вычтем одно из другого. Получим

$$
n-m+\sum_{i=1}^{j} \frac{c_{i}}{\chi\left(s_{1}\right) \chi\left(s_{2}\right) \cdots \chi\left(s_{i}\right)} .
$$

Так как ряд из формулировки леммы 4.3 расходится, то существует $k \in \mathbb{N}$ такое, что $\forall l \geqslant k$

$$
n-m+\sum_{i=1}^{l} \frac{c_{i}}{\chi\left(s_{1}\right) \chi\left(s_{2}\right) \cdots \chi\left(s_{i}\right)}>0 .
$$

Пусть объект $X \in \widehat{\mathfrak{D}}_{\infty}^{\prime}$ есть пара последовательностей

$$
X=\left(\left\{n_{i}\right\}_{i=1}^{\infty},\left\{d_{i} \mid d_{i}=\left(s_{i}, c_{i}\right) \in \mathbf{D}_{\infty}=S_{\infty} \ltimes \mathbb{Z}_{\geqslant 0}\right\}_{i=1}^{\infty}\right)
$$

Пусть задан морфизм

$$
u=\left(\left\{t_{i} \mid t_{i} \in S_{\infty}\right\}_{i=1}^{\infty},\left\{k_{i} \mid k_{i} \in \mathbb{N}\right\}_{i=1}^{\infty}\right) \in \operatorname{Hom}_{\widehat{\mathfrak{D}}_{S_{\infty}}}\left(C_{\infty}\left(X^{\prime}\right), C_{\infty}(X)\right)
$$


Так как ряд

$$
\sum_{j=i}^{\infty} \frac{c_{j}}{\chi\left(s_{1}\right) \chi\left(s_{2}\right) \cdots \chi\left(s_{i}\right)}
$$

расходится для любого $i \geqslant 1$, то тогда по лемме 4.3 сушествует $l_{i}>k_{i}$ такое, что

$$
n_{i} * t_{i} * s_{k_{i}+1} * \cdots * s_{l_{i}} \leqslant n_{k_{i}} * d_{k_{i}+1} * \cdots * d_{l_{i}}
$$

Следовательно, последовательность

$$
\begin{array}{r}
f=\left\{f_{i} \mid f_{i}=\left(t_{i} * s_{k_{i}+1} * \cdots * s_{l_{i}}, n_{k_{i}} * d_{k_{i}+1} * \cdots * d_{l_{i}}-n_{i} * t_{i} * s_{k_{i}+1} * \cdots * s_{l_{i}}\right)\right. \\
\left.\in \operatorname{Hom}_{\mathfrak{D}}\left(n_{i}, n_{l_{i}+1}\right)\right\}_{i=1}^{\infty}
\end{array}
$$

задает морфизм в категории $\widehat{\mathfrak{D}}_{\infty}$ такой, что $C_{\infty}(f)=u$.

Пункт б) доказывается аналогично.

УТВЕРЖДЕНИЕ 4.5. Пусть $P$ - это счетная коммутативная сократимая nолугруппа. Тогда в категории $\widehat{\mathfrak{D}}_{P}$ существует обвект $X$ такой, что

$$
\operatorname{Hom}_{\widehat{\mathfrak{D}}_{P}}(X, X)=Q(P)
$$

ДокАЗАТЕльство. Пусть объект $X$ задается последовательностью $\left\{p_{i} \mid\right.$ $\left.p_{i} \in P\right\}_{i=1}^{\infty}$ такой, что для любого элемента $p \in P$ сушествует бесконечное число индексов $i \in \mathbb{N}$ таких, что $p_{i}=p$.

Из утверждения 4.1 следует, что существует естественное вложение $\widehat{\rho}$ полугруппы $\operatorname{Hom}_{\widehat{\mathfrak{D}}_{P}}(X, X)$ в группу $Q(P)$. Докажем, что это вложение является сюръекцией.

Пусть $a / b \in Q(P)$. По определению последовательности $\left\{p_{i} \mid p_{i} \in P\right\}_{i=1}^{\infty}$ сушествует такое $k_{1} \in \mathbb{N}$, что $p_{k_{1}-1}=b$. Тогда положим $f_{1}=p_{1} * p_{2} * \cdots * p_{k_{1}-2} * a$. Опять по определению последовательности $\left\{p_{i} \mid p_{i} \in P\right\}_{i=1}^{\infty}$ существует такое $k_{2}>k_{1}$, что $p_{k_{2}-1}=p_{1}$. Тогда положим $f_{2}=p_{1} * p_{2} * p_{3} * \cdots * p_{k_{1}-2} * a * p_{k_{1}} *$ $\cdots * p_{k_{2}-2}$. Опять существует такое $k_{3}>k_{2}$, что $p_{k_{3}-1}=p_{2}$. Тогда положим

$$
f_{3}=p_{1} * p_{2} * p_{3} * \cdots * p_{k_{1}-2} * a * p_{k_{1}} * \cdots * p_{k_{2}-2} * p_{k_{2}} * \cdots * p_{k_{3}-2} .
$$

Продолжая индуктивно, мы построим пару последовательностей

$$
f=\left(\left\{f_{i} \mid f_{i} \in P\right\}_{i=1}^{\infty},\left\{k_{i} \mid k_{i} \in \mathbb{N}\right\}_{i=1}^{\infty}\right)
$$

которая задает морфизм объекта $X$ в категории $\widehat{\mathfrak{D}}_{P}$ и $\widehat{\rho}(f)=a / b$.

Из этого утверждения и утверждения 4.3 сразу следует, что существуют диагональные алгебры Ли, группа внешних автоморфизмов которых есть $\mathbb{Z}_{2} \times \mathbb{Z}^{\infty}$ или $\mathbb{Z}^{\infty}$. 
Для построения дальнейших примеров воспользуемся еше раз утверждением 2.6 . Выделим в полугруппе $S_{\infty}$ подполугруппу пар $(a, b)$ таких, что $a b=0$, и обозначим ее через $R$. Ясно, что подполугруппа $R$ обладает следующим свойством: если $r_{1}, r_{2} \in P$ и $r_{1} * r_{2} \in R$, то $r_{1}, r_{2} \in R$. Следовательно, если $X=\left\{r_{i} \mid r_{i} \in\right.$ $\left.R \subset S_{\infty}\right\}_{i=1}^{\infty}$, тo $\operatorname{Aut}_{\widehat{\mathfrak{D}}_{S_{\infty}}}(X)=\operatorname{Aut}_{\widehat{\mathfrak{D}}_{R}}(X)$.

Полугруппа $R$ содержит полугруппу натуральных чисел $\mathbb{N}$ по умножению в качестве подполугрупшы $N$ пар вида $(a, 0)$, а также и группу $\mathbb{Z}_{2}$ в качестве подмножества пар $\{(1,0),(0,1)\}$. Ясно, что полугруппа $R$ изоморфна прямому произведению $\mathbb{Z}_{2} \times N$. Отсюда вытекает, что если $X=\left\{r_{i} \mid r_{i} \in N \subset R\right\}_{i=1}^{\infty}$, то $\operatorname{Aut}_{\widehat{\mathfrak{D}}_{R}}(X)=\mathbb{Z}_{2} \times \operatorname{Aut}_{\widehat{\mathfrak{D}}_{N}}(X)$.

Полугруппа $N$ изоморфна полугруппе натуральных чисел $\mathbb{N}$ по умножению. Зафиксируем простые натуральные числа $p_{1}, \ldots, p_{k}$ в количестве $k$ штук. Обозначим через $N\left(p_{1}, \ldots, p_{k}\right)$ полугруппу натуральных чисел $n$ таких, что $n=p_{1}^{\alpha_{1}} \cdots p_{k}^{\alpha_{k}}$. Ясно, что если $n_{1}, n_{2} \in \mathbb{N}$ и $n_{1} n_{2} \in N\left(p_{1}, \ldots, p_{k}\right)$, то $n_{1}, n_{2} \in N\left(p_{1}, \ldots, p_{k}\right)$. Следовательно, опять применяя утверждение 2.6, получаем, что если $X=\left\{n_{i} \mid n_{i} \in\right.$

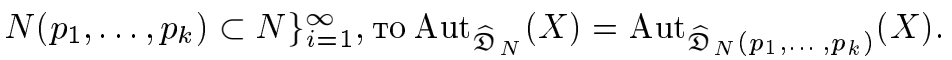

Так как $Q\left(N\left(p_{1}, \ldots, p_{k}\right)\right)$ есть $\mathbb{Z}^{k}$, то из этого рассуждения и утверждения 4.5 следует, что сушествуют диагональные алгебры Ли, у которых группа внешних автоморфизмов есть $\mathbb{Z}_{2} \times \mathbb{Z}^{k}$, где $k$ любое.

Напомним, что полугруппа $S_{0}$ изоморфна полугрупше $\mathbb{N}_{\text {even }}$ четных натуральных чисел. Опять зафиксируем простые натуральные числа $p_{1}, \ldots, p_{k}$ в количестве $k$ штук и определим полугруппу $S_{0}\left(p_{1}, \ldots, p_{k}\right)$ четных натуральных чисел $n$ таких, что $n=2^{\alpha_{0}} p_{1}^{\alpha_{1}} \cdots p_{k}^{\alpha_{k}}$. Так как $Q\left(S_{0}\left(p_{1}, \ldots, p_{k}\right)\right)=\mathbb{Z}^{k+1}$, то точно так же показывается, что существуют диагональные алгебры Ли, у которых группа внешних автоморфизмов есть $\mathbb{Z}^{k}$, где $k \geqslant 1$.

Для полноты картины приведем пример, показьвающий, что сушествует диагональная алгебра Ли, у которой группа внешних автоморфизмов тривиальна. Для этого занумеруем простые нечетные числа, например, в порядке возрастания. И определим объект $X=\left\{2 p_{i}\right\}_{i=1}^{\infty}$ категории $\widehat{\mathfrak{D}}_{S_{0}}$. Пусть пара $f=\left(\left\{f_{i}\right\}_{i=1}^{\infty},\left\{k_{i}\right\}_{i=1}^{\infty}\right)$ задает морфизм объекта $X$. Предположим, что $k_{1}>1$, тогда из того, что $f$-морфизм, следуют равенства

$$
2^{k_{i}-k_{1}-1} p_{k_{1}} \cdots p_{k_{i}-1} f_{1}=f_{i} 2^{i-1} p_{1} \cdots p_{i-1}
$$

для любого $i \geqslant 2$. Из этих равенств вытекает, что число $f_{1}$ должно делится на $p_{i-1}$ для любого $i \geqslant 2$. Следовательно, $k_{1}=1$. Рассуждая аналогично, получаем, что $k_{i}=i$ для любого $i \geqslant 1$. Из полученного в соответствии с определением категории $\widehat{\mathfrak{D}}_{S_{0}}$ легко выводится, что

$$
\text { Aut } \widehat{\mathfrak{D}}_{S_{0}}(X)=\langle e\rangle \text {. }
$$

В заключение мне бы хотелось поблагодарить моего научного руководителя Ю.А. Бахтурина, по настоятельной просьбе которого была написана эта статья, за проявленный интерес к работе. 


\section{Список литературы}

1. Бахтурин Ю. А., ШІтраде Г. Локально конечномерные простые алгебры Ли // Матем. сб. 1994. Т. 185 . № 2. C. 3-32.

2. Винберг Э.Б., Онищик А. Л. Семинар по группам Ли и алгебраическим группам. М.: Наука, 1988.

3. Baranov A.A. Diagonal localle finite Lie algebras // Preprint. Inst. Math. Acad. Sci. Belarus. V. 3 (515), 1996.

4. Yanson I. A., Zhdanovich D. V. The set of the direct limits of Lie algebras of the type A // Communications in algebra. 1996. V. 24. № 23. P. 1125-1156.

5. Гельфанд С.И., Манин Ю.И. Методы гомологической алгебры. М.: Наука, 1988.

6. Понтрязин Л. С. Непрерьвные группт. М.: Наука, 1984.

Московский государственный

Поступила в редакцию университет им. М.В. Ломоносова 